\title{
OSCILLATORITY OF NONLINEAR SYSTEMS WITH STATIC FEEDBACK*
}

\author{
DENIS V. EFIMOV ${ }^{\dagger}$ AND ALEXANDER L. FRADKOV $\ddagger$
}

\begin{abstract}
New Lyapunov-like conditions for oscillatority of dynamical systems in the sense of Yakubovich are proposed. Unlike previous results these conditions are applicable to nonlinear systems and allow for consideration of nonperiodic, e.g., chaotic modes. Upper and lower bounds for oscillations amplitude are obtained. The relation between the oscillatority bounds and excitability indices for the systems with the input are established. Control design procedure providing nonlinear systems with oscillatority property is proposed. Examples illustrating proposed results for Van der Pol system, Lorenz system, and Hindmarsh-Rose neuron model as well as computer simulation results are given.
\end{abstract}

Key words. analysis of oscillations, control of oscillations

AMS subject classifications. 34C15, 93B52

DOI. $10.1137 / 070706963$

1. Introduction. Most works on analysis or synthesis of nonlinear systems are devoted to studying stability-like behavior. Their typical results show that the motions of a system are close to a certain limit motion (limit mode) that either exists in the system or it is created by a controller. Evaluating deflection of the system trajectory from the limit mode, one may obtain quantitative information about system behavior [10, 27].

During recent years an interest in studying more complex dynamical systems behavior including oscillatory and, particularly, chaotic modes has grown significantly. Most authors deal with relaxed stability properties (orbital stability, Zhukovsky stability, partial stability) of some periodic limit modes $[16,19]$. However, in order to study irregular, chaotic behavior the development of analysis and design methods for nonperiodical oscillations is needed. One such method based on the concept of excitability index (limit oscillation amplitude) for the systems excited with a bounded control was proposed in $[7,8]$.

It is worth noting that there exist many definitions for the term "oscillation" $[11,16]$. For example, oscillation is understood as "any effect that varies in a backand-forth or reciprocating manner" [6]. Otherwise, oscillation is the behavior of a sequence or a function, that does not converge, but also does not diverge to $+\infty$ or $-\infty$; that is, oscillation is the failure to have a limit [29]. Geometrically, an oscillating function of real numbers follows some path in a space, without settling into eversmaller regions. In more simple cases the path might look like a loop coming back on itself, that is, periodic behavior; in more complex cases it may be a quite irregular movement covering a whole region [29]. Existing approaches based on Lyapunov stability theory $[17,23]$ or relaxed stability properties (orbital stability, Zhukovsky

* Received by the editors October 31, 2007; accepted for publication (in revised form) October 13, 2008; published electronically February 13, 2009.

http://www.siam.org/journals/sicon/48-2/70696.html

†Systems and Control, Université de Liège, Bat. 28, B-4000 Liege Sart-Tilman, Belgium (efimov@ montefiore.ulg.ac.be) and Control of Complex Systems Laboratory, Institute of Problem of Mechanical Engineering, Bolshoi av., 61, V.O., St-Petersburg, 199178 Russia.

$\ddagger$ Control of Complex Systems Laboratory, Institute of Problem of Mechanical Engineering, Bolshoi av., 61, V.O., St-Petersburg, 199178 Russia (alf@control.ipme.ru). 
stability, partial stability) $[16,19,24]$ are not completely suitable for study of complex oscillations. Indeed, these approaches require information on some limit modes, which stability should be investigated (that is not suitable for chaotic or irregular oscillations, for example). Besides, these approaches are not suitable for distinguishing between simple bounded behavior and oscillating one (a trajectory can converge to a steadystate solution that is a stable behavior from any kind of stability definition, but it is not an oscillation). Despite significant success in study of regular oscillations $[4,5,12,18,20]$, comprehensive solutions for generic irregular oscillations have not been obtained yet.

An important and useful concept for studying irregular oscillations is that of "oscillatority" introduced by V.A.Yakubovich in 1973 [31]. Frequency domain conditions for oscillatority were obtained for Lurie systems, and split in linear and nonlinear parts $[16,31,32]$. However, when studying physical and biological systems in many cases it is hard to decompose the system into two parts: Linear nominal system plus nonlinear feddback. Mechanical systems (where energy plays a role of Lyapunov function) serve as a widespread example of such systems. Extension of analysis and design methods to oscillations in such class of systems is still to appear.

In this paper an approach to detection of oscillations and design of oscillatory systems for a class of nonlinear systems is suggested. New conditions for oscillatority of dynamical systems in the sense of Yakubovich are proposed. These conditions are applicable to nonlinear systems, and they are formulated in terms of Lyapunov functions existence. As a result upper and lower bounds for oscillations amplitude are obtained. A variant of converse Lyapunov theorem for strictly unstable systems is proposed. The relation between the oscillatority bounds and excitability indices for the systems with input are established. Design procedure for oscillations excitation is presented. Potentiality of the proposed technique is illustrated by four examples of analytical computations and computer simulations.

The main advantage of the obtained solution consists in possibility of application to a wide range of oscillation analysis and design problems. The proposed conditions are applicable even in the cases when other existing solutions cannot be used due to complexity of oscillations or system models $[5,18,20]$.

Section 2 contains auxiliary statements and definitions (two preliminary results are placed in Appendix). Main definitions and oscillation existence conditions are presented in section 3. Section 4 deals with the task of static feedback design, which ensures oscillations appearance in closed loop system with desired bounds on amplitude. Conclusion is given in section 5. Examples illustrating proposed results for Van der Pol system, Lorenz system, and Hindmarsh-Rose neuron model as well as computer simulation results are presented in the text.

2. Preliminaries. Let us consider a general model of nonlinear dynamical system:

$$
\dot{\mathbf{x}}=\mathbf{f}(\mathbf{x}, \mathbf{u}) ; \quad \mathbf{y}=\mathbf{h}(\mathbf{x}),
$$

where $\mathbf{x} \in R^{n}$ is the state space vector; $\mathbf{u} \in R^{m}$ is the input vector; $\mathbf{y} \in R^{p}$ is the output vector; $\mathbf{f}$ and $\mathbf{h}$ are locally Lipschitz continuous functions on $R^{n}, \mathbf{h}(0)=0$, and $\mathbf{f}(0,0)=0$. For initial condition $\mathbf{x}_{0} \in R^{n}$ and Lebesgue measurable input $\mathbf{u}$ the solution $\mathbf{x}\left(\mathbf{x}_{0}, \mathbf{u}, t\right)$ of the system (1) is defined at least locally for $t \leq T$, $\mathbf{y}\left(\mathbf{x}_{0}, \mathbf{u}, t\right)=\mathbf{h}\left(\mathbf{x}\left(\mathbf{x}_{0}, \mathbf{u}, t\right)\right)$ (further we will simply write $\mathbf{x}(t)$ or $\mathbf{y}(t)$ if all other arguments are clear from the context). If for all initial conditions $\mathbf{x}_{0} \in R^{n}$ and inputs $\mathbf{u}$ the solutions are defined for all $t \geq 0$, then such system is called forward complete. 
In this work we will consider feedback connection of system (1) with static system $\mathbf{u}=\mathbf{k}(\mathbf{y})$.

As usual, it is said that a continuous function $\rho: R_{+} \rightarrow R_{+}$belongs to class $K$, if it is strictly increasing and $\rho(0)=0 ; \rho \in K_{\infty}$ if $\rho \in K$ and $\rho(s) \rightarrow \infty$ for $s \rightarrow \infty$; Lebesgue measurable function $\mathrm{x}: R_{+} \rightarrow R^{n}$ is essentially bounded, if $\|\mathbf{x}\|=e s s \sup \{|\mathbf{x}(t)|, t \geq 0\}<+\infty$, where $|\cdot|$ denotes usual Euclidean norm, $R_{+}=\{\tau \in R: \tau \geq 0\}$. Notation $D V(\mathbf{x}) \mathbf{F}(\cdot)$ stands for directional derivative of function $V$ with respect to vector field $\mathbf{F}$ if function $V$ is differentiable and for Dini derivative in the direction of $\mathbf{F}$

$$
D V(\mathbf{x}) \mathbf{F}(\cdot)=\lim _{t \rightarrow 0^{+}} \inf \frac{V(\mathbf{x}+t \mathbf{F}(\cdot))-V(\mathbf{x})}{t}
$$

if function $V$ is Lipschitz continuous. In what follows we need the standard dissipativity property [30] and some its modifications. Function $f\left(x_{1}, \ldots, x_{n}\right)$ defined on $R^{n}$ is called monotone if the condition $x_{1} \leq x_{1}^{\prime}, \ldots, x_{n} \leq x_{n}^{\prime}$ implies that everywhere either $f\left(x_{1}, \ldots, x_{n}\right) \leq f\left(x_{1}^{\prime}, \ldots, x_{n}^{\prime}\right)$ or $f\left(x_{1}, \ldots, x_{n}\right) \geq f\left(x_{1}^{\prime}, \ldots, x_{n}^{\prime}\right)$ everywhere.

DEFINITION 1. The system (1) is dissipative if there exists continuous function $V: R^{n} \rightarrow R_{+}$and a function $\varpi: R^{n+m+p} \rightarrow R$ such that for all $\mathbf{x}_{0} \in R^{n}$ and Lebesgue measurable and locally essentially bounded $\mathbf{u}: R_{+} \rightarrow R^{m}$ the following inequality is satisfied:

$$
V(\mathbf{x}(t)) \leq V\left(\mathbf{x}_{0}\right)+\int_{0}^{t} \varpi(\mathbf{x}(\tau), \mathbf{y}(\tau), \mathbf{u}(\tau)) d \tau, \quad t \geq 0 .
$$

The functions $\varpi$ and $V$ are called supply rate and storage functions of the system (1).

In the case when storage function is continuously differentiable, inequality (2) can be rewritten in a simple form:

$$
\dot{V}(\mathbf{x}, \mathbf{u})=L_{\mathbf{f}(\mathbf{x}, \mathbf{u})} V(\mathbf{x}) \leq \varpi(\mathbf{x}, \mathbf{u}, \mathbf{y}) .
$$

Definition 2. Dissipative system (1) is called

- passive if $\varpi(\mathbf{x}, \mathbf{y}, \mathbf{u})=\mathbf{y}^{T} \mathbf{u}-\beta(\mathbf{x})$, where $\beta$ is a continuous function reflecting the dissipation rate in the system; if $\beta(\mathbf{x}) \geq \widehat{\beta}(|\mathbf{x}|), \widehat{\beta} \in K$, then system (1) is called strictly passive [13];

- h-dissipative, if it has continuously differentiable storage function $V$ and

$$
\begin{gathered}
\underline{\alpha}(|\mathbf{y}|) \leq V(\mathbf{x}) \leq \bar{\alpha}(|\mathbf{x}|), \quad \omega(\mathbf{y}, \mathbf{u})=-\alpha(|\mathbf{y}|)+\sigma(|\mathbf{u}|), \\
\sigma \in K, \quad \alpha, \underline{\alpha}, \bar{\alpha} \in K_{\infty} ;
\end{gathered}
$$

- input-output-to-state stable (IOSS), if it has continuously differentiable storage function $W$ and $[26]$

$$
\begin{gathered}
\alpha_{1}(|\mathbf{x}|) \leq W(\mathbf{x}) \leq \alpha_{2}(|\mathbf{x}|), \quad \alpha_{1}, \alpha_{2} \in K_{\infty} \\
\omega(\mathbf{x}, \mathbf{y}, \mathbf{u})=-\alpha_{3}(|\mathbf{x}|)+\sigma_{1}(|\mathbf{u}|)+\sigma_{2}(|\mathbf{y}|),
\end{gathered}
$$

$\alpha_{3} \in K_{\infty}, \quad \sigma_{1}, \sigma_{2} \in K[26] ;$

- input-to-state stable (ISS), if it has continuously differentiable storage function $U$ and $[21]$

$$
\begin{gathered}
\alpha_{4}(|\mathbf{x}|) \leq U(\mathbf{x}) \leq \alpha_{5}(|\mathbf{x}|), \quad \alpha_{4}, \alpha_{5} \in K_{\infty} ; \\
\omega(\mathbf{x}, \mathbf{y}, \mathbf{u})=-\alpha_{6}(|\mathbf{x}|)+\delta(|\mathbf{u}|), \quad \alpha_{6} \in K_{\infty}, \quad \delta \in K .
\end{gathered}
$$

Copyright (c) by SIAM. Unauthorized reproduction of this article is prohibited. 
If inequality sign in (2) for the case $\varpi(\mathbf{x}, \mathbf{y}, \mathbf{u})=\mathbf{y}^{T} \mathbf{u}-\beta(\mathbf{x})$ can be replaced with equality, then it is said that the system possesses passivity property with known dissipation rate $\beta$.

Term $h$-dissipativity was introduced with minor differences in [2]. An important example of such kind of systems is $\mathbf{y}$-strictly passive systems [13]. Also, passive system (1) can be transformed to $h$-dissipative under suitable feedback transformation.

Storage functions for IOSS and ISS systems are called Lyapunov functions [23, 26]. Existence of corresponding Lyapunov functions is the equivalent characterization of ISS and IOSS properties [21, 26].

The interrelations of the properties introduced in Definition 2 are established in the Lemma A.1 (see Appendix), which was proved in [1] with a more restrictive requirement for $h$-dissipativity storage function:

$$
\alpha_{7}(|\mathbf{x}|) \leq V(\mathbf{x}) \leq \alpha_{8}(|\mathbf{x}|), \quad \alpha_{7}, \alpha_{8} \in K_{\infty} .
$$

General result in this direction was obtained in [15], where it was proven that inputto-output stability (this property is closely connected with $h$-dissipativity; see also [24] for more details) and IOSS are equivalent to ISS property for the system (1).

3. Oscillatority conditions. At first it is necessary to give a precise definition of the term "oscillatority" placed in the title of this section and the paper. There are several approaches to define oscillation phenomena for nonlinear dynamical systems [16]. Perhaps, the most general one is the concept introduced by Yakubovich [31, 32]. Here we recover definitions from [31,32] with some mild modifications [11, 16] dealing with high dimension and general form of the system.

Definition 3. Solution $\mathbf{x}\left(\mathbf{x}_{0}, 0, t\right)$ with $\mathbf{x}_{0} \in R^{n}$ of system (1) is called $\left[\pi^{-}, \pi^{+}\right]$oscillation with respect to output $\psi=\eta(\mathbf{x})$ (where $\eta: R^{n} \rightarrow R$ is a continuous monotone function) if the solution is defined for all $t \geq 0$ and

$$
\varliminf_{t \rightarrow+\infty} \psi(t)=\pi^{-} ; \quad \varlimsup_{t \rightarrow+\infty} \psi(t)=\pi^{+} ; \quad-\infty<\pi^{-}<\pi^{+}<+\infty .
$$

Solution $\mathbf{x}\left(\mathbf{x}_{0}, 0, t\right)$ with $\mathbf{x}_{0} \in R^{n}$ of system (1) is called oscillating, if there exist some output $\psi$ and constants $\pi^{-}, \pi^{+}$such that $\mathbf{x}\left(\mathbf{x}_{0}, 0, t\right)$ is $\left[\pi^{-}, \pi^{+}\right]$-oscillation with respect to the output $\psi$. Forward complete system (1) with $\mathbf{u}(t) \equiv 0, t \geq 0$ is called oscillatory, if for almost all $\mathbf{x}_{0} \in R^{n}$ solutions of the system $\mathbf{x}\left(\mathbf{x}_{0}, 0, t\right)$ are oscillating. Oscillatory system (1) is called uniformly oscillatory, if for almost all $\mathbf{x}_{0} \in R^{n}$ for corresponding solutions $\mathbf{x}\left(\mathbf{x}_{0}, 0, t\right)$ there exist output $\psi$ and constants $\pi^{-}, \pi^{+}$not depending on initial conditions.

In other words, the solution $\mathbf{x}\left(\mathbf{x}_{0}, 0, t\right)$ is oscillating if output $\psi(t)=\eta\left(\mathbf{x}\left(\mathbf{x}_{0}, 0, t\right)\right)$ is asymptotically bounded and there is no single limit value of $\psi(t)$ for $t \rightarrow+\infty$ that is close to definition of oscillatority from [29].

Note that the term "almost all solutions" is used to emphasize that generally system (1) for $\mathbf{u}(t) \equiv 0, t \geq 0$ has a nonempty set of equilibrium points; thus, there exists a set of initial conditions with zero measure such that corresponding solutions are not oscillations. It is worth stressing that constants $\pi^{-}$and $\pi^{+}$are exact asymptotic bounds for output $\psi$. Therefore, in order to compute these values the exact estimates for the system solutions should be known, which is a hard task for general nonlinear system (1). Fortunately, information on approximate estimates of constants $\pi^{-}$and $\pi^{+}$is sufficient to obtain estimates on system amplitude oscillations. The oscillation property introduced in Definition 3 is defined for zero input and any initial conditions of system (1). The following property is a closely related characterization 
of the system behavior, which develops the proposed above property for the case of nonzero input but for specified initial conditions [8].

Definition 4. Let $\mathbf{u}: R_{+} \rightarrow R^{m}$ be Lebesgue measurable and essentially bounded function and $\mathbf{x}_{0} \in R^{n}$ be given such that $\mathbf{x}\left(\mathbf{x}_{0}, \mathbf{u}, t\right)$ be defined for all $t \geq 0$. The functions $\chi_{\psi, \mathbf{x}_{0}}^{-}(\gamma), \chi_{\psi, \mathbf{x}_{0}}^{+}(\gamma)$ defined for $\|\mathbf{u}\| \leq \gamma, \gamma \in R_{+}$are called lower and upper excitation indices of system (1) in point $\mathbf{x}_{0}$ with respect to the output $\psi=\eta(\mathbf{x})$ (where $\eta: R^{n} \rightarrow R$ is a continuous monotone function), if

$$
\begin{gathered}
\left(\chi_{\psi, \mathbf{x}_{0}}^{-}(\gamma), \chi_{\psi, \mathbf{x}_{0}}^{+}(\gamma)\right)=\underset{(a, b) \in E(\gamma)}{\arg \max }\{b-a\} \\
E(\gamma)=\left\{(a, b):\left(\begin{array}{c}
a=\varliminf_{1 \rightarrow+\infty} \eta\left(\mathbf{x}\left(\mathbf{x}_{0}, \mathbf{u}, t\right)\right), \\
b=\varlimsup_{t \rightarrow+\infty} \eta\left(\mathbf{x}\left(\mathbf{x}_{0}, \mathbf{u}, t\right)\right)
\end{array}\right)\right\}_{\|\mathbf{u}\| \leq \gamma} .
\end{gathered}
$$

Lower and upper excitation indices of a forward complete system (1) with respect to the output $\psi$ are

$$
\chi_{\psi}^{-}(\gamma)=\inf _{\mathbf{x}_{0} \in R^{n}} \chi_{\psi, \mathbf{x}_{0}}^{-}(\gamma), \quad \chi_{\psi}^{+}(\gamma)=\sup _{\mathbf{x}_{0} \in R^{n}} \chi_{\psi, \mathbf{x}_{0}}^{+}(\gamma) .
$$

In the same way it is possible to introduce indices for a vector output $\psi=\eta(\mathbf{x})$, in this case indices would be vectors of the same dimension as the output $\psi$.

Excitation indices characterize ability of system (1) to exhibit forced or controllable oscillations caused by bounded inputs. It is clear that properties $\pi^{-}=\chi_{\psi}^{-}(0)$ and $\pi^{+}=\chi_{\psi}^{+}(0)$ are satisfied. For nonzero inputs the excitability indices characterize maximum (over specified set of inputs $\|\mathbf{u}\| \leq \gamma$ ) asymptotic amplitudes $\chi_{\psi}^{+}(\gamma)-\chi_{\psi}^{-}(\gamma)$ of $\psi$.

Note that it is useful to calculate or estimate values of $\chi_{\psi}^{-}(\gamma)$ and $\chi_{\psi}^{+}(\gamma)$ for all $0 \leq \gamma<+\infty$ due to the following reason. Let oscillation amplitude be an inverse function of input amplitude, then the maximum oscillation amplitude be reached for some $\gamma *$ and for all $\gamma \geq \gamma *$ the amplitude decreases. The indices $\chi_{\psi}^{-}(\gamma)$ and $\chi_{\psi}^{+}(\gamma)$ preserve their values for $\gamma \geq \gamma *$. Hence, to catch the critical value $\gamma *$ of input amplitude providing maximum output amplitude for $\psi$, it is necessary to build full graphics of functions $\chi_{\psi}^{-}(\gamma)$ and $\chi_{\psi}^{+}(\gamma)$. The obtained characteristics will be closely related with the Cauchy gain recently investigated in [22] (in fact, $\pi^{+}-\pi^{-}$or $\chi_{\psi, \mathbf{x}_{0}}^{+}(\gamma)-\chi_{\psi, \mathbf{x}_{0}}^{-}(\gamma)$ are asymptotic amplitudes of $\psi(t)$ in the sense of [22] for zero or nonzero input $\mathbf{u}$, while $\chi_{\psi}^{+}(\gamma)$ reflects the Cauchy gain of the system (1)).

On the other hand, excitation indices from Definition 4 describe robustness of the oscillations property proposed in Definition 3. Conditions of oscillations existence in the system are summarized in the following theorem.

Theorem 1. Let system (1) with $\mathbf{u}(t) \equiv 0, t \in R_{+}$, i.e.,

$$
\dot{\mathbf{x}}=\mathbf{f}(\mathbf{x}, 0),
$$

have two continuous and locally Lipschitz Lyapunov functions $V_{1}$ and $V_{2}$ satisfying for all $\mathbf{x} \in R^{n}$ the following inequalities:

$$
\begin{aligned}
& v_{1}(|\mathbf{x}|) \leq V_{1}(\mathbf{x}) \leq v_{2}(|\mathbf{x}|), \quad v_{3}(|\mathbf{x}|) \leq V_{2}(\mathbf{x}) \leq v_{4}(|\mathbf{x}|), \quad v_{1}, v_{2}, v_{3}, v_{4} \in K_{\infty} \\
& \text { and for some } 0<X_{1}<v_{1}^{-1} \circ v_{2} \circ v_{3}^{-1} \circ v_{4}\left(X_{2}\right)<+\infty: \\
& D V_{1}(\mathbf{x}) \mathbf{f}(\mathbf{x}, 0)>0 \text { for } 0<|\mathbf{x}|<X_{1} \text { and } \mathbf{x} \notin \Xi \\
& D V_{2}(\mathbf{x}) \mathbf{f}(\mathbf{x}, 0)<0 \text { for }|\mathbf{x}|>X_{2} \text { and } \mathbf{x} \notin \Xi
\end{aligned}
$$

Copyright $@$ by SIAM. Unauthorized reproduction of this article is prohibited. 
where $\Xi \subset R^{n}$ is a set with zero Lebesgue measure, which contain all equilibriums of the system, and

$$
\Omega \cap \Xi=\emptyset,
$$

where $\Omega=\left\{\mathbf{x}: v_{2}^{-1} \circ v_{1}\left(X_{1}\right)<|\mathbf{x}|<v_{3}^{-1} \circ v_{4}\left(X_{2}\right)\right\}$.

Then the system (3) is oscillatory.

Proof. Consider set $\Xi_{0} \subset R^{n}$ of initial conditions not containing equilibrium points (which belong to set $\Xi$ ) of system (3). Then the solutions of the system starting from $\Xi_{0}$ are globally bounded, due to $\dot{V}_{2}<0$ for $|\mathbf{x}|>X_{2}$, and defined for all $t \geq 0$. Since the trajectory $\mathbf{x}\left(\mathbf{x}_{0}, 0, t\right), \mathbf{x}_{0} \in \Xi_{0}, t \geq 0$ is bounded, it has a nonempty closed, invariant, and compact $\omega$-limit set, which belongs to the set $\Omega$. Indeed, $V_{2}(t)$ asymptotically enters into the set where $V_{2}(t)<v_{4}\left(X_{2}\right)$, then $|\mathbf{x}(t)|<v_{3}^{-1} \circ v_{4}\left(X_{2}\right)$. In the same way function $V_{1}(t)$ is upper bounded and its limit values fall into the set where $V_{1}(t)>v_{1}\left(X_{1}\right)$; i.e., again $|\mathbf{x}(t)|>v_{2}^{-1} \circ v_{1}\left(X_{1}\right)$.

As it was supposed, $\Omega$ does not contain equilibrium points of the system. Hence, $\omega$-limit set also does not include such invariant solutions. Then for each $\mathbf{x}_{0} \in \Xi_{0}$ there exists an index $i, 1 \leq i \leq n$ such that the solution is $\left[\pi^{-}, \pi^{+}\right]$-oscillation with respect to output $x_{i}$ with $-v_{3}^{-1} \circ v_{4}\left(X_{2}\right) \leq \pi^{-}<\pi^{+}<v_{3}^{-1} \circ v_{4}\left(X_{2}\right)$. Suppose that there is no such output. It means that for all $1 \leq i \leq n$ for output $x_{i}$ equality $\pi^{-}=\pi^{+}$holds. However, the latter could be true only in equilibrium points, which are excluded from the set $\Omega$ by the theorem conditions. Therefore, for almost all initial conditions the system solutions have such oscillating output and system (3) is oscillatory by Definition 3. Note that for different $\mathbf{x}_{0} \in \Xi_{0}$ oscillating outputs $x_{i}$ may exist for different $i, 1 \leq i \leq n$.

Remark 1 . The set $\Omega$ determines lower and upper bounds for the values of $\pi^{-}$ and $\pi^{+}$.

Like in [32] one can consider the Lyapunov function candidate for linearized near the origin system (3) as a function $V_{1}$ to prove local instability of the system. Instead of existence of storage function $V_{2}$, one can require just boundedness of the system solution $\mathbf{x}(t)$ with a known upper bound. It can be obtained using another approach not dealing with time derivative of Lyapunov function analysis. In this case Theorem 1 is transforming into Theorem 3.4 from [11]; see also [33].

Corollary 1. Define $\Xi$ as the set of the system (3) equilibriums, i.e., $\Xi=\{\mathbf{x} \in$ $\left.R^{n}: \mathbf{f}(\mathbf{x}, 0)=0\right\}$, which consists in isolated points, and $\mathbf{A}\left(\mathbf{x}_{0}\right)=d \mathbf{f}(\mathbf{x}, 0) /\left.d \mathbf{x}\right|_{\mathbf{x}=\mathbf{x}_{0}}$ is the matrix of the system (3) linearization in point $\mathbf{x}_{0} \in R^{n}$. Let the following conditions be valid:

1. For all $\mathbf{x}_{0} \in \Xi$ the matrices of the system (3) linearization $\mathbf{A}\left(\mathbf{x}_{0}\right)$ have eigenvalues with positive real parts.

2. There exists $R>0$ such that for almost all initial conditions $\mathbf{x}_{0} \in R^{n}$ :

$$
\lim _{t \rightarrow+\infty}\left|\mathbf{x}\left(\mathbf{x}_{0}, 0, t\right)\right| \leq R
$$

Then the system (3) is oscillatory.

Proof. By conditions of the corollary for almost all initial conditions the $\omega$-limit set is compact and it does not contain the equilibriums of the system. Further the proof is similar to the proof of Theorem 1 .

Conditions of Theorem 1 are rather general and define the class of systems, which oscillatory behavior can be investigated by the approach, namely systems which have an attracting compact set in state space containing oscillatory movements of the 
systems. For such systems Theorem 1 or Corollary 1 give useful tools for testing their oscillating behavior and obtaining estimates for amplitude of oscillations.

Theorem 1 presents the sufficient conditions for system (1) to be oscillating in the sense of Yakubovich. It is possible to show that for a subclass of uniformly oscillating systems these conditions are also necessary. To prove this result we need the following two lemmas.

LEMma 1. Let there exist constant $r>0$ such that for solutions of systems (3) the following property is satisfied:

$$
0<\left|\mathbf{x}_{0}\right|<r \Rightarrow\left|\mathbf{x}\left(\mathbf{x}_{0}, 0, t\right)\right|>r
$$

for all $t \geq T_{\mathbf{x}_{0}}$, where $0<T_{\mathbf{x}_{0}}<+\infty$. Then there exists a continuous and locally Lipschitz-Lyapunov function $V_{1}(\mathbf{x})$ such that for all $\mathbf{x} \in R^{n}$

$$
v_{1}(|\mathbf{x}|) \leq V_{1}(\mathbf{x}) \leq v_{2}(|\mathbf{x}|), \quad v_{1}, v_{2} \in K_{\infty},
$$

additionally for all $0<|\mathbf{x}|<r$ it holds:

$$
D V_{1}(\mathbf{x}) \mathbf{f}(\mathbf{x}, 0)>0 .
$$

Proof. For $\left|\mathbf{x}_{0}\right|<r$ let us introduce the function:

$$
v\left(\mathbf{x}_{0}\right)=\inf _{0 \leq t \leq T_{\mathbf{x}_{0}}}\left|\mathbf{x}\left(\mathbf{x}_{0}, 0, t\right)\right| .
$$

According to conditions of the lemma this function admits the following properties:

(i) $v(0)=0$ and $v(\mathbf{x})>0$ for $0<|\mathbf{x}|<r$;

(ii) $v\left(\mathbf{x}_{0}\right)=\inf _{0 \leq t \leq T_{\mathbf{x}_{0}}+\Delta}\left|\mathbf{x}\left(\mathbf{x}_{0}, 0, t\right)\right|$ for any $\Delta \geq 0$.

Additionally for $0<|\mathbf{x}|<r$ the property $|v(0)-v(\mathbf{x})|=v(\mathbf{x}) \leq|\mathbf{x}|=|0-\mathbf{x}|$ holds, which means continuity of function $v$ at the origin. In the set $|\mathbf{x}|<r$ the relation $\delta(|\mathbf{x}|) \leq v(\mathbf{x}) \leq|\mathbf{x}|$ holds, where $\delta(s)=s(1+s)^{-1} \inf _{|\mathbf{x}|=s} v(\mathbf{x})$ is a continuous and strictly increasing function, $\delta(0)=0$. The locally Lipschitz property of function $v$ in the set $0<|\mathbf{x}|<r$ follows from the following series of inequalities satisfied for any $\mathbf{x}_{1}, \mathbf{x}_{2}$ belonging to this set and some constants $L>0, M>0$, $T=\max \left\{T_{\mathbf{x}_{1}}, T_{\mathbf{x}_{2}}\right\}$ :

$$
\begin{gathered}
\left|\mathbf{x}\left(\mathbf{x}_{1}, 0, t\right)-\mathbf{x}\left(\mathbf{x}_{2}, 0, t\right)\right| \leq M\left|\mathbf{x}_{1}-\mathbf{x}_{2}\right|, \quad t \leq T ; \\
|| \mathbf{x}\left(\mathbf{x}_{1}, 0, t\right)|-| \mathbf{x}\left(\mathbf{x}_{2}, 0, t\right) \| \leq L\left|\mathbf{x}_{1}-\mathbf{x}_{2}\right|, \quad t \leq T ; \\
\left|v\left(\mathbf{x}_{1}\right)-v\left(\mathbf{x}_{2}\right)\right|=\left|\inf _{0 \leq t \leq T}\right| \mathbf{x}\left(\mathbf{x}_{1}, 0, t\right)\left|-\inf _{0 \leq t \leq T}\right| \mathbf{x}\left(\mathbf{x}_{2}, 0, t\right) \| \\
\leq \sup _{0 \leq t \leq T}|| \mathbf{x}\left(\mathbf{x}_{1}, 0, t\right)|-| \mathbf{x}\left(\mathbf{x}_{2}, 0, t\right)|| \leq L\left|\mathbf{x}_{1}-\mathbf{x}_{2}\right| .
\end{gathered}
$$

By construction for initial conditions $\left|\mathbf{x}_{0}\right|<r$ the relation $v\left(\mathbf{x}\left(\mathbf{x}_{0}, 0, t\right)\right) \geq v\left(\mathbf{x}\left(\mathbf{x}_{0}\right.\right.$, $0,0)), t \leq T_{\mathbf{x}_{0}}$ holds, then $D v(\mathbf{x}) \mathbf{f}(\mathbf{x}, 0) \geq 0$ for all $|\mathbf{x}|<r$ and function $v(t)$ is not decreasing. To design a strictly increasing function let us introduce for $\left|\mathbf{x}_{0}\right|<r$ the function:

$$
V_{1}\left(\mathbf{x}_{0}\right)=\inf _{0 \leq t \leq T_{\mathbf{x}_{0}}} k(t) v\left(\mathbf{x}\left(\mathbf{x}_{0}, 0, t\right)\right)
$$

where $k: R_{+} \rightarrow R_{+}$is a continuously differentiable function with the following properties for all $t \in R_{+}$:

$$
\kappa_{1} \leq k(t) \leq \kappa_{2}, \quad 0<\kappa_{1}<\kappa_{2}<+\infty ; \quad \partial k / \partial t<0 .
$$

Copyright (c) by SIAM. Unauthorized reproduction of this article is prohibited. 
As an example of such function $k$ it is possible to choose the following one:

$$
k(t)=\kappa_{1}+\left(\kappa_{2}-\kappa_{1}\right) e^{-t}, \quad \dot{k}(t)=\left(\kappa_{1}-\kappa_{2}\right) e^{-t} .
$$

By construction $V_{1}(0)=0$ and $V_{1}(\mathbf{x})>0$ for $0<|\mathbf{x}|<r$. In the set $|\mathbf{x}|<r$ the relation $\kappa_{1} \delta(|\mathbf{x}|) \leq v(\mathbf{x}) \leq \kappa_{2}|\mathbf{x}|$ holds. The locally Lipschitz continuity of function $V_{1}$ in the set $0<|\mathbf{x}|<r$ follows from the same arguments, since the following series of inequalities are satisfied for any $\mathbf{x}_{1}, \mathbf{x}_{2}$ belonging to this set and some constants $L>0, M>0, T=\max \left\{T_{\mathbf{x}_{1}}, T_{\mathbf{x}_{2}}\right\}$ :

$$
\begin{aligned}
& \left|\mathbf{x}\left(\mathbf{x}_{1}, 0, t\right)-\mathbf{x}\left(\mathbf{x}_{2}, 0, t\right)\right| \leq M\left|\mathbf{x}_{1}-\mathbf{x}_{2}\right|, \quad t \leq T \\
& \left|v\left(\mathbf{x}_{1}\right)-v\left(\mathbf{x}_{2}\right)\right| \leq L\left|\mathbf{x}_{1}-\mathbf{x}_{2}\right| ; \\
& \left|v\left(\mathbf{x}\left(\mathbf{x}_{1}, 0, t\right)\right)-v\left(\mathbf{x}\left(\mathbf{x}_{2}, 0, t\right)\right)\right| \leq M L\left|\mathbf{x}_{1}-\mathbf{x}_{2}\right|, \quad t \leq T ; \\
& \left|V_{1}\left(\mathbf{x}_{1}\right)-V_{1}\left(\mathbf{x}_{2}\right)\right|=\left|\inf _{0 \leq t \leq T_{\mathbf{x}_{1}}} k(t) v\left(\mathbf{x}\left(\mathbf{x}_{1}, 0, t\right)\right)-\inf _{0 \leq t \leq T_{\mathbf{x}_{2}}} k(t) v\left(\mathbf{x}\left(\mathbf{x}_{2}, 0, t\right)\right)\right| \\
& \leq \sup _{0 \leq t \leq T} k(t) \mid v\left(\mathbf{x}\left(\mathbf{x}_{1}, 0, t\right)-v\left(\mathbf{x}\left(\mathbf{x}_{2}, 0, t\right)\left|\leq \kappa_{2} M L\right| \mathbf{x}_{1}-\mathbf{x}_{2} \mid .\right.\right.
\end{aligned}
$$

For $|\mathbf{x}| \geq r$ extend function $V_{1}: R^{n} \rightarrow R_{+}$in such a way that for all $\mathbf{x} \in R^{n}$ function $V_{1}$ is continuous and locally Lipschitz and there exist two functions $v_{1}, v_{2} \in K_{\infty}$ such that for all $\mathrm{x} \in R^{n}$ :

$$
v_{1}(|\mathbf{x}|) \leq V_{1}(\mathbf{x}) \leq v_{2}(|\mathbf{x}|)
$$

where $v_{1}(s) \leq \kappa_{1} \delta(s), \kappa_{2} s \leq v_{2}(s)$ for $s<r$. By construction for initial conditions $0<\left|\mathbf{x}_{0}\right|<r$ the following relations hold:

$$
\begin{aligned}
& V_{1}\left(\mathbf{x}\left(\mathbf{x}_{0}, 0, t\right)\right)=\inf _{0 \leq \tau \leq T_{\mathbf{x}\left(\mathbf{x}_{0}, 0, t\right)}} k(\tau) v\left(\mathbf{x}\left[\mathbf{x}\left(\mathbf{x}_{0}, 0, t\right), 0, \tau\right]\right) \\
& >\inf _{0 \leq \tau \leq T_{\mathbf{x}_{0}}} k(\tau) v\left(\mathbf{x}\left[\mathbf{x}_{0}, 0, \tau\right]\right)=V_{1}\left(\mathbf{x}_{0}\right), 0<t \leq T_{\mathbf{x}_{0}}, T_{\mathbf{x}\left(\mathbf{x}_{0}, 0, t\right)}<T_{\mathbf{x}_{0}},
\end{aligned}
$$

then $D V_{1}(\mathbf{x}) \mathbf{f}(\mathbf{x}, 0)>0$ for all $0<|\mathbf{x}|<r$.

Under conditions of Lemma 1 solutions $\mathbf{x}\left(\mathbf{x}_{0}, 0, t\right)$ of the system (3) are locally unstable for initial conditions $\mathbf{x}_{0}$ which belong to the sphere $0<\left|\mathbf{x}_{0}\right|<r$. According to the result of the lemma in this case the system (3) has corresponding Lyapunov function with positive time derivative for $0<|\mathbf{x}|<r$. It is possible to say that Lemma 1 presents a variant of necessary conditions of a Lyapunov function existence for a subclass of strictly unstable systems, which is a new result.

Lemma 2. Let there exist constants $R>0$ and $0<T_{R, \mathbf{x}_{0}}<+\infty$ such that for solutions of the system (3) the following property is satisfied:

$$
\left|\mathbf{x}_{0}\right|>R \Rightarrow\left|\mathbf{x}\left(\mathbf{x}_{0}, 0, t\right)\right|<R, t \geq T_{R, \mathbf{x}_{0}} .
$$

Then there exists a continuous and locally Lipschitz-Lyapunov function $V_{2}(\mathbf{x})$ such that for all $\mathbf{x} \in R^{n}$

$$
v_{3}(|\mathbf{x}|) \leq V_{2}(\mathbf{x}) \leq v_{4}(|\mathbf{x}|), \quad v_{3}, v_{4} \in K_{\infty},
$$

and for all $|\mathbf{x}|>R$ it holds that

$$
D V_{2}(\mathbf{x}) \mathbf{f}(\mathbf{x}, 0)<0
$$

Copyright (C) by SIAM. Unauthorized reproduction of this article is prohibited. 
Proof. For $\left|\mathbf{x}_{0}\right|>R$ let us introduce the function

$$
v\left(\mathbf{x}_{0}\right)=\sup _{t \geq 0}\left|\mathbf{x}\left(\mathbf{x}_{0}, 0, t\right)\right|=\sup _{T_{R, \mathbf{x}_{0} \geq t \geq 0}}\left|\mathbf{x}\left(\mathbf{x}_{0}, 0, t\right)\right| .
$$

Under conditions of the lemma the property $v(\mathbf{x})>R$ for $|\mathbf{x}|>R$ is satisfied. Additionally due to continuity of solutions of the system (3) with respect to initial conditions for each $\varepsilon>0$ there exists $\delta>0$ such that

$\mathbf{x}_{1} \in R^{n}, \quad \mathbf{x}_{2} \in R^{n}$

$\left|\mathbf{x}_{1}-\mathbf{x}_{2}\right| \leq \delta \quad \Rightarrow\left|\mathbf{x}\left(\mathbf{x}_{2}, 0, t\right)-\mathbf{x}\left(\mathbf{x}_{1}, 0, t\right)\right| \leq \varepsilon, t \leq t_{\max }, t_{\max }=\max \left\{T_{R, \mathbf{x}_{1}}, T_{R, \mathbf{x}_{2}}\right\}$.

Note that for solutions of the system the equality $\sup _{t_{\max } \geq t \geq 0}\left|\mathbf{x}\left(\mathbf{x}_{i}, 0, t\right)\right|=\sup _{t \geq 0} \mid$ $\mathbf{x}\left(\mathbf{x}_{i}, 0, t\right) \mid, i=1,2$ is satisfied. Then for any initial conditions under constrain $\mid \mathbf{x}_{1}-$ $\mathbf{x}_{2}|\leq \delta,| \mathbf{x}_{1}|>R,| \mathbf{x}_{2} \mid>R$ it holds that

$$
\begin{aligned}
& \left|v\left(\mathbf{x}_{1}\right)-v\left(\mathbf{x}_{2}\right)\right| \\
& \quad=\left|\sup _{t_{\max } \geq t \geq 0}\right| \mathbf{x}\left(\mathbf{x}_{1}, 0, t\right)\left|-\sup _{t_{\max } \geq t \geq 0}\right| \mathbf{x}\left(\mathbf{x}_{2}, 0, t\right)|| \\
& \quad \leq \sup _{t_{\max } \geq t \geq 0}\left|\mathbf{x}\left(\mathbf{x}_{1}, 0, t\right)\right|-\left|\mathbf{x}\left(\mathbf{x}_{2}, 0, t\right)\right| \mid \leq \varepsilon,
\end{aligned}
$$

which means continuity of function $v$ for $|\mathbf{x}|>R$. In the set $|\mathbf{x}|>R$ for function $v$ the following relation also holds:

$$
|\mathbf{x}| \leq v(\mathbf{x}) \leq \delta(|\mathbf{x}|)
$$

where $\delta(s)=s+\sup _{|\mathbf{x}|=s} v(\mathbf{x})$ is a continuous and strictly increasing function. The locally Lipschitz continuity of function $v$ into set $|\mathbf{x}|>R$ follows from the series of inequalities satisfied for any $\mathbf{x}_{1}, \mathbf{x}_{2}$ from the set and some $L>0$ :

$$
\begin{aligned}
& || \mathbf{x}\left(\mathbf{x}_{1}, 0, t\right)|-| \mathbf{x}\left(\mathbf{x}_{2}, 0, t\right)|| \leq L\left|\mathbf{x}_{1}-\mathbf{x}_{2}\right|, \quad t \leq t_{\max }, \\
& \quad=\left|v\left(\mathbf{x}_{1}\right)-v\left(\mathbf{x}_{2}\right)\right| \\
& \quad \leq \sup _{t_{\max } \geq t \geq 0}\left|\mathbf{x}\left(\mathbf{x}_{1}, 0, t\right)\right|-\sup _{t_{\max } \geq t \geq 0}\left|\mathbf{x}\left(\mathbf{x}_{2}, 0, t\right)\right| \mid
\end{aligned}
$$

By construction for all initial conditions with $\left|\mathbf{x}_{0}\right|>R$ it holds that

$$
v(t)=v\left(\mathbf{x}\left(\mathbf{x}_{0}, 0, t\right)\right) \leq v\left(\mathbf{x}\left(\mathbf{x}_{0}, 0,0\right)\right)=v(0),
$$

then $D v(\mathbf{x}) \mathbf{f}(\mathbf{x}, 0) \leq 0$ for $|\mathbf{x}|>R$ and function $v$ is not increasing. To design a strictly decreasing function, consider the following one for $\left|\mathbf{x}_{0}\right|>R$ :

$$
V_{2}\left(\mathbf{x}_{0}\right)=\sup _{T_{R, \mathbf{x}_{0}} \geq t \geq 0} k(t) v\left(\mathbf{x}\left(\mathbf{x}_{0}, 0, t\right)\right),
$$

where $k: R_{+} \rightarrow R_{+}$is a continuously differentiable function with properties for all $t \in R_{+}$:

$$
\kappa_{3} \leq k(t) \leq \kappa_{4}, \quad 0<\kappa_{3}<\kappa_{4}<+\infty ; \quad \partial k / \partial t>0
$$

Copyright $@$ by SIAM. Unauthorized reproduction of this article is prohibited. 
For example, it is possible to choose as a function $k(t)$ the following one:

$$
k(t)=\frac{\kappa_{3}+\kappa_{4} t}{1+t}, \quad \dot{k}(t)=\frac{\kappa_{4}-\kappa_{3}}{(1+t)^{2}} .
$$

Under conditions of the lemma in the set $|\mathbf{x}|>R$ for function $V_{2}$ the relation $\kappa_{3}|\mathbf{x}| \leq$ $V_{2}(\mathbf{x}) \leq \kappa_{4} \delta(|\mathbf{x}|)$ holds. For any initial conditions under constrain $\left|\mathbf{x}_{1}-\mathbf{x}_{2}\right| \leq \bar{\delta}$, $\left|\mathbf{x}_{1}\right|>R,\left|\mathbf{x}_{2}\right|>R$ it holds that

$$
\begin{aligned}
& \left|V_{2}\left(\mathbf{x}_{1}\right)-V_{2}\left(\mathbf{x}_{2}\right)\right| \\
& \quad=\left|\sup _{T_{R, \mathbf{x}_{1} \geq t \geq 0}} k(t) v\left(\mathbf{x}\left(\mathbf{x}_{1}, 0, t\right)\right)-\sup _{T_{R, \mathbf{x}_{2} \geq t \geq 0}} k(t) v\left(\mathbf{x}\left(\mathbf{x}_{2}, 0, t\right)\right)\right| \\
& \quad \leq \sup _{t_{\max } \geq t \geq 0} k(t)|| \mathbf{x}\left(\mathbf{x}_{1}, 0, t\right)|-| \mathbf{x}\left(\mathbf{x}_{2}, 0, t\right) \mid \leq \kappa_{4} \varepsilon,
\end{aligned}
$$

which means continuity of function $V_{2}$ for $|\mathbf{x}|>R$. The locally Lipschitz continuity of function $V_{2}$ into set $|\mathbf{x}|>R$ follows from the same inequalities satisfied for any $\mathbf{x}_{1}, \mathbf{x}_{2}$ from the set and some $L>0$ :

$$
\begin{aligned}
& \left|V_{2}\left(\mathbf{x}_{1}\right)-V_{2}\left(\mathbf{x}_{2}\right)\right| \\
& \quad=\left|\sup _{T_{R, \mathbf{x}_{1} \geq t \geq 0}} k(t) v\left(\mathbf{x}\left(\mathbf{x}_{1}, 0, t\right)\right)-\sup _{T_{R, \mathbf{x}_{2} \geq t \geq 0} k(t) v\left(\mathbf{x}\left(\mathbf{x}_{2}, 0, t\right)\right) \mid} k(t)\right|\left|\mathbf{x}\left(\mathbf{x}_{1}, 0, t\right)\right|-\left|\mathbf{x}\left(\mathbf{x}_{2}, 0, t\right)\right|\left|\leq \kappa_{4} L\right| \mathbf{x}_{1}-\mathbf{x}_{2} \mid .
\end{aligned}
$$

For $|\mathbf{x}| \leq R$ we extend the definition of function $V_{2}$ such that for all $\mathbf{x} \in R^{n}$ function $V_{2}: R^{n} \rightarrow R_{+}$would be continuous and locally Lipschitz and for all $\mathrm{x} \in R^{n}$ :

$$
v_{3}(|\mathbf{x}|) \leq V_{2}(\mathbf{x}, t) \leq v_{4}(|\mathbf{x}|),
$$

where $v_{3}, v_{4} \in K_{\infty}$ and $\kappa_{4} s \geq v_{3}(s), v_{4}(s) \geq \kappa_{3} \delta(s)$ for $s>R$. By construction for all initial conditions with $\left|\mathbf{x}_{0}\right|>R$, it holds that

$$
\begin{aligned}
& V_{2}(t)=V_{2}\left(\mathbf{x}\left(\mathbf{x}_{0}, 0, t\right)\right)=\sup _{T_{R, \mathbf{x}\left(\mathbf{x}_{0}, 0, t\right) \geq \tau \geq 0} k(\tau) v\left(\mathbf{x}\left[\mathbf{x}\left(\mathbf{x}_{0}, 0, t\right), 0, \tau\right]\right)} k\left(\mathbf{x}_{0}\right)=V_{2}(0), 0<t \leq T_{R, \mathbf{x}_{0}}, T_{R, \mathbf{x}\left(\mathbf{x}_{0}, 0, t\right)}<T_{R, \mathbf{x}_{0}}, \\
& \quad<\sup _{T_{R, \mathbf{x}_{0}} \geq \tau \geq 0} k(\tau) v\left(\mathbf{x}\left[\mathbf{x}_{0}, 0, \tau\right]\right)=V_{2}\left(\mathbf{x}_{0}\right)
\end{aligned}
$$

and then $D V_{2}(\mathbf{x}) \mathbf{f}(\mathbf{x}, 0)<0$ for $|\mathbf{x}|>R$.

Under conditions of the lemma set, $A=\{\mathbf{x}:|\mathbf{x}|<R\}$ is a globally attractive invariant set for solutions of system (3) with zero input; see also [17] for other converse Lyapunov theorems for set stability. Contrarily to the case considered in this paper, the Lyapunov functions $W: R^{n} \rightarrow R_{+}$proposed in [17] possess for all $\mathbf{x} \in R^{n}$ the properties

$$
\alpha_{1}\left(|\mathbf{x}|_{A}\right) \leq W(\mathbf{x}) \leq \alpha_{2}\left(|\mathbf{x}|_{A}\right), \quad \alpha_{1}, \alpha_{2} \in K_{\infty},
$$

where $|\mathbf{x}|_{A}$ is the distance from point $\mathbf{x}$ to the set $A$, which stability is investigated.

Now we are ready to substantiate the necessary conditions of oscillatority.

THEOREM 2. Let system (3) be uniformly oscillatory with respect to the output $\psi=\eta(\mathbf{x})$ (where $\eta: R^{n} \rightarrow R$ is a continuous function), and for all $\mathbf{x} \in R^{n}$ the following relations are satisfied:

$$
\chi_{1}(|\mathbf{x}|) \leq \eta(\mathbf{x}) \leq \chi_{2}(|\mathbf{x}|), \quad \chi_{1}, \chi_{2} \in K_{\infty} ;
$$

Copyright (C) by SIAM. Unauthorized reproduction of this article is prohibited. 
the set of initial conditions for which the system is not oscillating consists in just one point $\Xi=\{\mathbf{x}: \mathbf{x}=0\}$. Then there exist two continuous and locally Lipschitz Lyapunov functions $V_{1}: R^{n} \rightarrow R_{+}$and $V_{2}: R^{n} \rightarrow R_{+}$such that for all $\mathbf{x} \in R^{n}$ the inequalities hold:

$$
\begin{gathered}
v_{1}(|\mathbf{x}|) \leq V_{1}(\mathbf{x}) \leq v_{2}(|\mathbf{x}|), \quad v_{3}(|\mathbf{x}|) \leq V_{2}(\mathbf{x}) \leq v_{4}(|\mathbf{x}|), \quad v_{1}, v_{2}, v_{3}, v_{4} \in K_{\infty} \\
D V_{1}(\mathbf{x}) \mathbf{f}(\mathbf{x}, 0)>0 \text { for } 0<|\mathbf{x}|<\chi_{2}^{-1}\left(\pi^{-}\right) \\
D V_{2}(\mathbf{x}) \mathbf{f}(\mathbf{x}, 0)<0 \text { for }|\mathbf{x}|>\chi_{1}^{-1}\left(\pi^{+}\right) .
\end{gathered}
$$

Proof. Since system (3) is uniformly oscillatory with respect to output $\psi=\eta(\mathbf{x})$, then for almost all initial conditions (except the origin) there exists constants $-\infty<$ $\pi^{-}<\pi^{+}<+\infty$ such that

$$
\begin{aligned}
& \varliminf_{t \rightarrow+\infty} \eta\left(\mathbf{x}\left(\mathbf{x}_{0}, 0, t\right)\right)=\varliminf_{t \rightarrow+\infty} \psi(t)=\pi^{-} ; \\
& \varlimsup_{t \rightarrow+\infty} \eta\left(\mathbf{x}\left(\mathbf{x}_{0}, 0, t\right)\right)=\varlimsup_{t \rightarrow+\infty} \psi(t)=\pi^{+} .
\end{aligned}
$$

By radial unboundedness and positive definiteness of function $\eta$ it means that all solutions of the system converge to the invariant set $\Omega=\left\{\mathbf{x}: \chi_{2}^{-1}\left(\pi^{-}\right) \leq \mathbf{x} \leq\right.$ $\left.\chi_{1}^{-1}\left(\pi^{+}\right)\right\}$. Then there exist constants $X_{1}<\chi_{2}^{-1}\left(\pi^{-}\right)$and $X_{2}>\chi_{1}^{-1}\left(\pi^{+}\right)$such that conditions of Lemmas 1 and 2 hold for $r=X_{1}$ and $R=X_{2}$. Based on these facts, the existence of Lyapunov functions $V_{1}$ and $V_{2}$ follows.

For uniformly oscillatory systems with single equilibrium point at the origin, Theorems 1 and 2 give necessary and sufficient conditions of oscillations existence (Van der Pol or Hindmarsh and Rose systems (see below) are examples of uniformly oscillatory systems). The oscillatority concept introduced by Yakubovich covers situations of periodic and chaotic oscillations. That allows one to analyze behavior of wide spectrum of oscillating dynamical systems using common approach. Note that for chaotic systems constants $\pi^{-}$and $\pi^{+}$evaluate geometrical size of strange attractor. Let us demonstrate on examples the efficiency of the proposed approach for analysis of oscillation phenomena in nonlinear systems.

Example 1. Consider the Van der Pol system:

$$
\dot{x}_{1}=x_{2} ; \quad \dot{x}_{2}=-x_{1}+\varepsilon\left(1-x_{1}^{2}\right) x_{2},
$$

where $\varepsilon>0$ some parameter. To detect presence of oscillations in this system, it is required (according to Theorem 1) to find two Lyapunov functions, which establish local instability of equilibrium $(0,0)$ and global boundedness of the system solutions. Since the system has only one equilibrium point in the origin, the set $\Omega$ from the theorem does not contain the point $(0,0)$. Let us consider the following Lyapunov functions for $0<\varepsilon \leq 1$ :

$$
\begin{gathered}
V_{1}(\mathbf{x})=0.5\left(\left(1-\varepsilon+\varepsilon^{-1}\right) x_{1}^{2}+\left(1+\varepsilon^{-1}\right) x_{2}^{2}+\varepsilon\left(x_{2}-\varepsilon x_{1}\right)^{2}\right) \\
V_{2}(\mathbf{x})=0.5\left(\varepsilon^{-1} x_{2}-2 x_{1}+1 / 3 x_{1}^{3}\right)^{2}+1 / 12 x_{1}^{4} \\
\dot{V}_{1}=\varepsilon x_{2}^{2}+\left(x_{2}-\varepsilon x_{1}\right)^{2}+\left[\varepsilon^{3} x_{1}-\left(1+\varepsilon+\varepsilon^{2}\right) x_{2}\right] x_{1}^{2} x_{2} \\
\dot{V}_{2}=-\left[0.5 \sqrt{\varepsilon}\left(2-\varepsilon^{-2}\right) x_{1}-\varepsilon^{-0.5} x_{2}\right]^{2}-1 / 3 \varepsilon^{-1} x_{1}^{4} \\
+\left[0.25 \varepsilon\left(2-\varepsilon^{-2}\right)^{2}+2 \varepsilon^{-1}\right] x_{1}^{2} .
\end{gathered}
$$

Copyright (c) by SIAM. Unauthorized reproduction of this article is prohibited. 


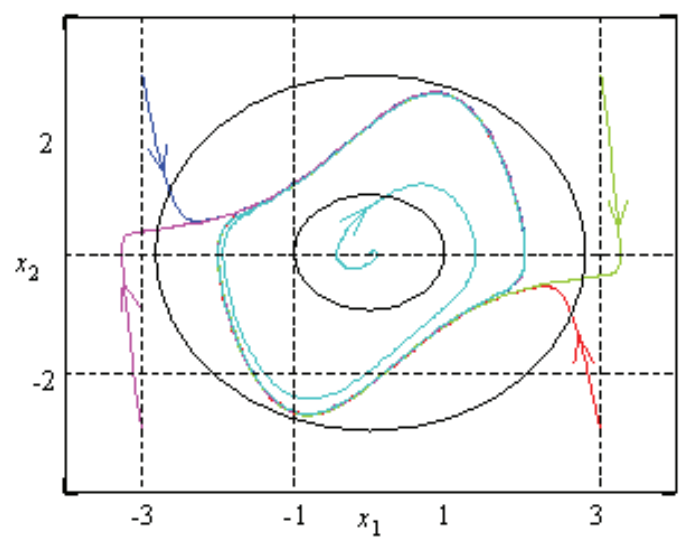

FIG. 1. Trajectories and set $\Omega$ for Van der Pol system.

Function $\dot{V}_{1}$ is strictly positive in the set $0<|\mathbf{x}|<X_{1}$, where $X_{1}=X_{1}(\varepsilon)>0$ (the same conclusion was obtained in [12] for $\varepsilon=1, X_{1}=\sqrt{3}$ ). Instability of the system also can be verified for a linearized version of the system, which eigenvalues $\lambda_{1,2}=0.5\left(\varepsilon \pm \sqrt{\varepsilon^{2}-4}\right)$ are always positive for $\varepsilon>0$. Analyzing function $\dot{V}_{2}$ it is possible to obtain $X_{2} \leq \sqrt{3\left[0.25 \varepsilon^{2}\left(2-\varepsilon^{-2}\right)^{2}+2\right]}$. Results of the set $\Omega$ calculation and computer simulation of the system for $\varepsilon=1$ are presented in Figure 1, where the set $\Omega$ is bounded by solid ellipses.

Example 2. Let us consider Lorenz model:

$$
\begin{aligned}
& \dot{x}=\sigma(y-x), \\
& \dot{y}=r x-y-x z, \\
& \dot{z}=-b z+x y,
\end{aligned}
$$

where parameters $\sigma=10, r=28$, and $b=8 / 3$. With such choice of parameter values the system is chaotic, which is a good example of complex nonlinear oscillation processes. To apply the result of Theorem 1 here let us note that the system has three equilibriums with coordinates

$$
\mathbf{x}_{e}^{1}=\left(\begin{array}{lll}
0 & 0 & 0
\end{array}\right)^{T}, \quad \mathbf{x}_{e}^{2}=(\sqrt{72} \sqrt{72} 27)^{T}, \quad \mathbf{x}_{e}^{3}=(-\sqrt{72}-\sqrt{72} 27)^{T} .
$$

The matrix of linear approximation of this system at the equilibriums

$$
A\left(\mathbf{x}_{e}\right)=\left[\begin{array}{lll}
-\sigma & \sigma & 0 \\
r-\mathbf{x}_{e, 3} & -1 & -\mathbf{x}_{e, 1} \\
\mathbf{x}_{e, 2} & \mathbf{x}_{e, 1} & -b
\end{array}\right]
$$

has for the given values of parameters eigenvalues with positive real parts for all equilibriums. Therefore the system is locally unstable. Lyapunov function

$$
V(x, y, z)=0.5\left(\sigma^{-1} x^{2}+y^{2}+(z-r)^{2}\right)
$$

for this system has the following time derivative:

$$
\begin{aligned}
\dot{V} & =-x^{2}+x y-y^{2}-b z^{2}+r b z \\
& \leq-0.5 x^{2}-0.5 y^{2}-0.5 b z^{2}+0.5 b r^{2},
\end{aligned}
$$

Copyright $@$ by SIAM. Unauthorized reproduction of this article is prohibited. 


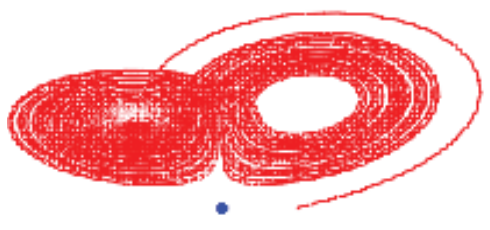

FIG. 2. Trajectory of Lorenz system.

which implies global boundedness of all trajectories of Lorenz system. All conditions of Corollary 1 are satisfied and system is oscillatory in the sense of Definition 3. An example of state space trajectory of the system is presented in Figure 2 (blue dots correspond to coordinates of equilibriums $\mathbf{x}_{e}^{i}$ ).

Example 3. A Hindmarsh and Rose model neuron is defined by the following system of differential equations [14]:

$$
\begin{aligned}
& \dot{x}=-a x^{3}+b x^{2}+y-z+u, \\
& \dot{y}=c-d x^{2}-y, \\
& \dot{z}=\varepsilon\left[s\left(x-x_{0}\right)-z\right],
\end{aligned}
$$

where $x \in R_{+}$is the membrane potential, $y \in R_{+}$is recovery variable, and $z \in R_{+}$is adaptation variable. External stimulation is given by input $u \in R$. It is a well-known fact that this model demonstrates complex oscillatory behavior for the following values of the model parameters $a=1, b=3, c=1, d=5, s=4, x_{0}=0.795, \varepsilon=0.001$ with input $u=0$. Let us investigate oscillatority property of the model for the case $u=0$ applying the proposed approach.

As the first let us compute the number of equilibriums in the system which coordinates are solutions of the following system of nonlinear equations:

$$
\begin{aligned}
& -a x_{e}^{3}+(b-d) x_{e}^{2}-s x_{e}+s x_{0}+c=0 ; \\
& y_{e}=c-d x_{e}^{2} ; \\
& z_{e}=s\left(x_{e}-x_{0}\right) .
\end{aligned}
$$

As in the first example we are interested in a situation when the model has a single equilibrium. This is the case when the first cubic equation above has only one real solution and two complex solutions. Under conditions

$$
\begin{gathered}
n \geq 0, \quad \frac{m}{6 a}+\frac{2}{3} \frac{3 s a-(b-d)^{2}}{a u} \neq 0, \\
n=4 s^{3} a-s^{2}(b-d)^{2}+\left[27 a^{2}\left(s x_{0}+c\right)-18 s a(b-d)+4(b-d)^{3}\right]\left(s x_{0}+c\right), \\
m=\sqrt[3]{12 a \sqrt{3 n}-36 s a(b-d)+108 a^{2}\left(s x_{0}+c\right)+8(b-d)^{3}},
\end{gathered}
$$

the model has the following single equilibrium

$$
\begin{aligned}
& x_{e}=a^{-1}\left(m / 6-2 / 3\left[3 s a-(b-d)^{2}\right] / m+(b-d) / 3\right) ; \\
& y_{e}=c-d x_{e}^{2} ; \\
& z_{e}=s\left(x_{e}-x_{0}\right) .
\end{aligned}
$$




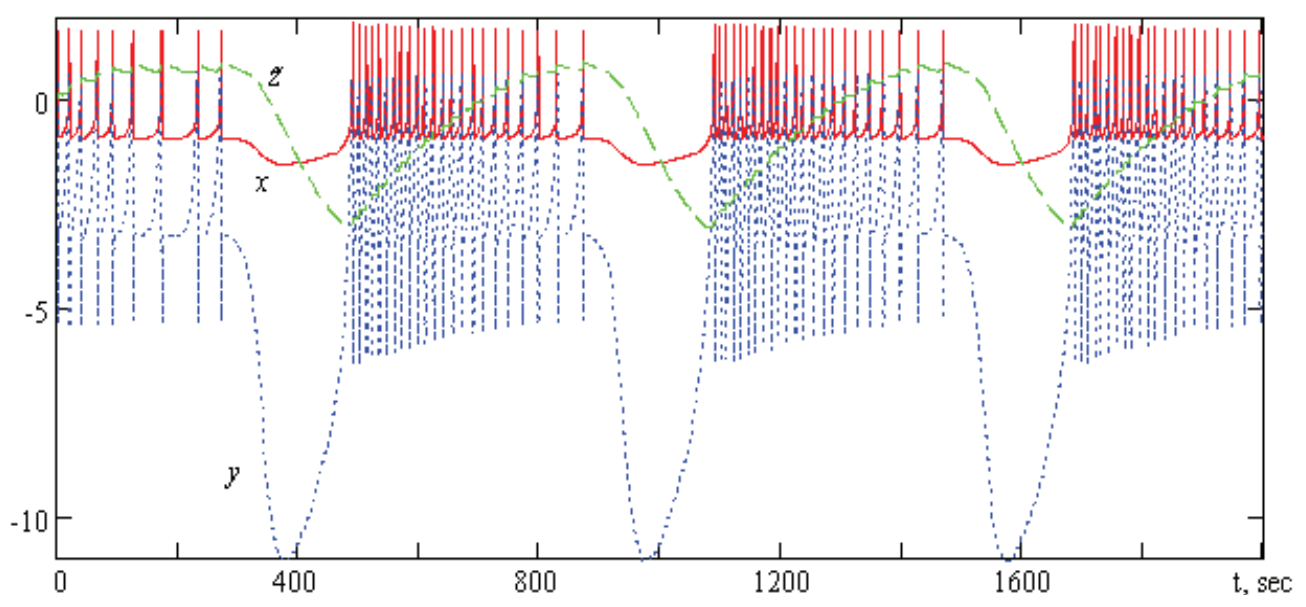

FIG. 3. Trajectories of Hindmarsh and Rose neuron model.

To prove global boundedness of the system solutions, it is possible to use the following Lyapunov function:

$$
V_{2}=0.5\left(s x^{2}+\varepsilon^{-1} z^{2}+s a y^{2} / d^{2}\right),
$$

in which the time derivative for the model admits inequality:

$\dot{V}_{2} \leq s x\left(-0.5 a x^{3}+b x^{2}+8 d^{2} x / a\right)-0.25 s a y^{2} / d^{2}-0.5 z^{2}+8 s a c^{2} / d^{2}+0.5 s^{2} x_{0}^{2}$.

To prove local instability of the equilibrium, consider linearization of the system with matrix

$$
A\left(x_{e}, y_{e}, z_{e}\right)=\left[\begin{array}{lll}
-3 a x_{e}^{2}+2 b x_{e} & 1 & -1 \\
-2 d x_{e} & -1 & 0 \\
\varepsilon s & 0 & -\varepsilon
\end{array}\right] .
$$

According to Hurwitz criteria matrix $A$ has eigenvalues with positive real parts if at least one from the following inequalities is satisfied:

$$
\begin{gathered}
3 a x_{e}^{2}-2 b x_{e}+1+\varepsilon \leq 0, \quad 3 a x_{e}^{2}+2(d-b) x_{e}+s \leq 0, \\
3 a(\varepsilon+1) x_{e}^{2}+2(d-(\varepsilon+1) b) x_{e}+\varepsilon(s+1) \leq 0, \\
9 a^{2}(\varepsilon+1) x_{e}^{4}+a[6 d-12(\varepsilon+1) b] x_{e}^{3}+\left[4 b[(\varepsilon+1) b-d]+3 a\left[\varepsilon^{2}+(2+s) \varepsilon+1\right]\right] x_{e}^{2} \\
+2\left[d-\left[\varepsilon^{2}+(s+2) \varepsilon+1\right] b\right] x_{e}+(s+1) \varepsilon^{2}+\varepsilon \leq 0 .
\end{gathered}
$$

Thus we obtain all set of restrictions on admissible values of the model parameters under which the system is uniformly oscillatory. The proposed values in [14] of the model parameters admit all these conditions (there exists single unstable equilibrium with globally bounded solutions). The result of the model simulation is shown in Figure 3, where $\tilde{z}=10 z$ is a scaled adaptation variable.

A link between oscillatority and excitation indices is established in the following corollary. 
Corollary 2. Let for initial condition $\mathbf{x}_{0} \in R^{n}$ the solution $\mathbf{x}\left(\mathbf{x}_{0}, \mathbf{k}(\mathbf{x}), t\right)$ of system (1) with control $\mathbf{u}=\mathbf{k}(\mathbf{x}), \mathbf{k}(0)=0$ be $\left[\pi^{-}, \pi^{+}\right]$-oscillation with respect to output

$$
\psi=\eta(\mathbf{x}), \quad \alpha_{1}(|\mathbf{x}|) \leq \eta(\mathbf{x}), \quad \alpha_{1} \in K_{\infty} .
$$

Then excitation indices of system (1) satisfy inequality

$$
\pi^{+}-\pi^{-} \leq \chi_{\psi, \mathbf{x}_{0}}^{+}(\gamma)-\chi_{\psi, \mathbf{x}_{0}}^{-}(\gamma),
$$

for $\gamma \geq \gamma *$, where $\gamma *=\sup _{|\mathbf{x}| \leq \alpha_{1}^{-1}\left(\pi^{+}\right)}|\mathbf{k}(\mathbf{x})|$.

Proof. From oscillatority property with respect to output $\psi$, the solutions of the closed by feedback $\mathbf{k}$ system (1) are asymptotically bounded:

$$
|\mathbf{x}(t)| \leq \alpha_{1}^{-1}\left(\pi^{+}\right), \quad t \geq 0 .
$$

Therefore input $\mathbf{u}=\mathbf{k}(\mathbf{x})$ is upper bounded by $\gamma \geq \gamma *$ and the statement follows from Definitions 3 and 4 (excitation indices are not decreasing functions of $\gamma$ ).

Hence, to compute estimates on excitation indices it is enough to find some control $\mathbf{k}$ for system (1), which ensures oscillations existence in closed loop system.

In the proof of Theorem 1 a component of state space vector was proposed as an oscillating output. However, such output does not discover all features of oscillation processes in the system and it does not restrict the possible set of oscillating variables of the system. To avoid this obstacle we formulate the same conclusion for output oscillations of system (3) rewriting conditions of the theorem with respect to $\mathbf{y}$ :

$$
\begin{gathered}
v_{1}(|\mathbf{y}|) \leq V_{1}(\mathbf{x}) \leq v_{2}(|\mathbf{y}|), v_{3}(|\mathbf{y}|) \leq V_{2}(\mathbf{x}) \leq v_{4}(|\mathbf{y}|), \\
D V_{1}(\mathbf{x}) \mathbf{f}(\mathbf{x}, 0)>0 \text { for } 0<|\mathbf{y}|<Y_{1} ; \\
D V_{1}(\mathbf{x}) \mathbf{f}(\mathbf{x}, 0)>0 \text { for }|\mathbf{y}|>Y_{2}, \\
Y_{1}<v_{1}^{-1} \circ v_{2} \circ v_{3}^{-1} \circ v_{4}\left(Y_{2}\right) .
\end{gathered}
$$

Then the set $\Omega=\left\{\mathbf{y}: v_{2}^{-1} \circ v_{1}\left(Y_{1}\right)<|\mathbf{y}|<v_{3}^{-1} \circ v_{4}\left(Y_{2}\right)\right\}$ and the system is oscillatory if set $\Omega$ does not contain equilibrium points of closed loop system $\dot{\mathbf{x}}=\mathbf{f}(\mathbf{x}, 0)$. A more constructive result, which points out on oscillating variables, can be presented as follows.

LEMMA 3. Let system (1) have IOSS Lyapunov function $W$ and h-dissipative storage function $V$ as in Definition 2 and $\lim _{s \rightarrow+\infty} \alpha(s)^{-1} \sigma_{2}(s)<+\infty$ (conditions of Lemma A.1 hold). Suppose that $\mathbf{u}=\mathbf{k}(\mathbf{x})$ and

(i) $\alpha_{6}(|\mathbf{x}|)>\delta(|\mathbf{k}(\mathbf{x})|)$ for $|\mathbf{x}|>X \geq 0$ and $\mathbf{x} \notin \Xi$,

(ii) $L_{\mathbf{f}(\mathbf{x}, \mathbf{k}(\mathbf{x}))} V(\mathbf{x})>0$ for $0<|\mathbf{h}(\mathbf{x})| \leq Y$ and $\mathbf{x} \notin \Xi$,

for some positive constants $X$ and $Y$ with $Y<\underline{\alpha}^{-1} \circ \bar{\alpha} \circ \alpha_{4}^{-1} \circ \alpha_{5}(X)$ (where functions $\alpha_{4}, \alpha_{5}, \alpha_{6}$ and $\delta$ defined in Lemma A.1), set $\Xi$ has zero Lebesgue measure. If set $\Omega=$ $\left\{V(\mathbf{x}): \underline{\alpha}(Y) \leq V(\mathbf{x}) \leq \bar{\alpha} \circ \alpha_{4}^{-1} \circ \alpha_{5}(X)\right\}$ does not contain equilibrium points of closed loop system $\dot{\mathbf{x}}=\mathbf{f}(\mathbf{x}, \mathbf{k}(\mathbf{x}))$, then the system is oscillatory.

Proof. First of all note that from point (i) the system satisfies all conditions from Lemma A.1 to be ISS with respect to input $\mathbf{u}$ and it also has bounded (i.e., defined for all $t \geq 0$ ) solutions due to property (i). As before, $\mathbf{x}(t)$ and $\mathbf{y}(t)$ have nonempty closed and compact $\omega$-limit sets, which are upper bounded by estimate $|\mathbf{x}| \leq \alpha_{4}^{-1} \circ \alpha_{5}(X)$. 
From point (ii) of the lemma it is possible to conclude that $\dot{V}>0$ for small enough $0<|\mathbf{y}| \leq Y$. Then the set of $\omega$-limit trajectories for function $V(t)$ belongs to the set $\Omega$. Now the result immediately follows similarly to the final steps of Theorem 1 proof.

Generically function $V$ depends on part of variables only, which helps to define a subset of oscillating variables in the system. Additionally, Lemma 3 points out a way to find functions $V_{1}$ and $V_{2}\left(V_{1}(\mathbf{x})=V(\mathbf{x})\right.$ and $V_{2}(\mathbf{x})=U(\mathbf{x})$ from Appendix). Results of proposed theorems and Lemma 3 do not deal with feedback $\mathbf{k}$ design problem. Now let us continue with the task of control design that ensures desired oscillation parameters for passive systems.

4. Stabilization of oscillation regimes. In this section the problem of feedback design for passive system is considered, and the proposed feedback ensures oscillatority of closed loop system. Section 4 is based on result of Lemma A.2, although conditions imposed on feedback $\mathbf{k}$ in the Lemma A.2 look complex and hardly verified, they are very natural and can be easily resolved. For example, if $\sigma_{1}$ and $\sigma_{2}$ are quadratic functions of their arguments, then control $\mathbf{k}$ with linear growth rate with respect to $\mathbf{y}$ satisfies all proposed conditions.

THEOREM 3. Let system (1) be passive with known dissipation rate $\beta$ and IOSS in the sense of Definition 2 and

$$
\underline{\alpha}(|\mathbf{y}|) \leq V(\mathbf{x}) \leq \bar{\alpha}(|\mathbf{x}|), \quad \underline{\alpha}, \bar{\alpha} \in K_{\infty} .
$$

Consider control $\mathbf{u}=\mathbf{k}(\mathbf{x})+\mathbf{d}$, which possesses the following properties for all $\mathbf{x} \in$ $R^{n}$ :

(1) for some $0<K<+\infty$,

$$
|\mathbf{k}(\mathbf{x})| \leq \lambda(|\mathbf{y}|)+K
$$

(2) decreasing of storage function $V$ for large values of the output, i.e., inequality holds

$$
\beta(\mathbf{x})-\mathbf{y}^{T} \mathbf{k}(\mathbf{x})+\mu(|\mathbf{d}|)+\mu(K) \geq \kappa(|\mathbf{y}|)+\mathbf{y}^{T} \mathbf{d} ;
$$

(3) $\mathbf{y}^{T} \mathbf{k}(\mathbf{x})>\beta(\mathbf{x})$ for $0<|\mathbf{y}|<Y<+\infty, Y<\underline{\alpha}^{-1} \circ \bar{\alpha} \circ \alpha_{4}^{-1} \circ \alpha_{5} \circ \alpha_{6}^{-1} \circ \delta(K)$, $\lim _{s \rightarrow+\infty} \frac{\sigma_{2}(s)+\sigma_{1} \circ \lambda(s)}{\kappa(s)}<+\infty$, where $\lambda \in K, \kappa \in K_{\infty}, \mu \in K$ (functions $\alpha_{4}, \alpha_{5}, \alpha_{6}$ and $\delta$ obtained in Lemma A.2) and $\mathbf{d} \in R^{m}$ is new input (Lebesgue measurable and essentially bounded function of time). Then

(i) system solutions are bounded;

(ii) if set $\Omega=\left\{V(\mathbf{x}): \underline{\alpha}(Y) \leq V(\mathbf{x}) \leq \bar{\alpha} \circ \alpha_{4}^{-1} \circ \alpha_{5} \circ \alpha_{6}^{-1} \circ \delta(K)\right\}$ does not contain equilibrium points of system $\dot{\mathbf{x}}=\mathbf{f}(\mathbf{x}, \mathbf{k}(\mathbf{x}))$ then for $\mathbf{d}(t) \equiv 0, t \geq 0$ closed loop system is an oscillatory one.

Proof. Introduce partition of control input:

$$
\mathbf{u}=\mathbf{k}(\mathbf{x})=-\mathbf{k}_{1}(\mathbf{x})+\mathbf{k}_{2}(\mathbf{x}),
$$

such that

$$
\begin{gathered}
\left|\mathbf{k}_{1}(\mathbf{x})\right| \leq \lambda(|\mathbf{y}|), \quad\left|\mathbf{k}_{2}(\mathbf{x})\right| \leq K \\
\mathbf{y}^{T} \mathbf{k}_{1}(\mathbf{x})+\beta(\mathbf{x})+\mu(|\mathbf{d}|) \geq \kappa(|\mathbf{y}|)+\mathbf{y}^{T} \mathbf{d} \\
\mathbf{y}^{T} \mathbf{k}_{2}(\mathbf{x})>\beta(\mathbf{x})+\mathbf{y}^{T} \mathbf{k}_{1}(\mathbf{x}) \text { for } 0<|\mathbf{y}|<Y<+\infty .
\end{gathered}
$$

Copyright (c) by SIAM. Unauthorized reproduction of this article is prohibited. 
This separation is possible due to conditions of Theorem 3. Introduce auxiliary input $\tilde{\mathbf{d}}=\mathbf{d}+\mathbf{k}_{2}(\mathbf{x})$ (essentially bounded by conditions of the theorem $\|\tilde{\mathbf{d}}\| \leq$ $K+\|\mathbf{d}\|)$. For system (1) all conditions of Lemma A.2 are satisfied for the feedback $\mathbf{u}=-\mathbf{k}_{1}(\mathbf{x})+\tilde{\mathbf{d}}$ and system is ISS with respect to input $\tilde{\mathbf{d}}$. According to ISS property [21] and boundedness of $\tilde{\mathbf{d}}$, boundedness of system solution immediately follows and statement (i) of Theorem 3 is proven. To justify statement (ii) note that the conditions of Lemma 3 also hold.

Theorem 3 extends the result from [3] and [28] to the case of general nonlinear dynamical systems. Additional special attention is given to the lower estimate of the oscillation amplitude for $\mathbf{d}(t) \equiv 0, t \geq 0$.

Exciting part $\mathbf{k}_{2}$ of feedback $\mathbf{k}$ defines the size of set $\Omega$ (due to constants $Y$ and $K$ are prescribed by $\mathbf{k}_{2}$ ) and, hence, it regulates the gap between values of $\pi^{-}$and $\pi^{+}$.

Remark 2. It is worth stressing that the control in Theorem 3 is proposed to satisfy some sector condition with respect to output $\mathbf{y}$. For design of such controls in practical application it is possible to use speed-gradient approach [9, 10], e.g., choose $\mathbf{u}=\varphi(\mathbf{y})$, where $\varphi(\mathbf{y})^{T} \mathbf{y}>0$ for $0<|\mathbf{y}|<Y_{1}$ and $\varphi(\mathbf{y})^{T} \mathbf{y}<0$ for $|\mathbf{y}|>Y_{2}>Y_{1}$.

Example 4. Let us consider controlled linear oscillator:

$$
\dot{x}_{1}=x_{2} ; \quad \dot{x}_{2}=-x_{1}+u,
$$

which is passive with storage function

$$
V(\mathbf{x})=0.5\left(x_{1}^{2}+x_{2}^{2}\right), \quad \dot{V}=x_{2} u,
$$

and IOSS with corresponding Lyapunov function

$$
\begin{gathered}
W(\mathbf{x})=0.5\left(x_{1}^{2}+\left(x_{1}+x_{2}\right)^{2}\right), \\
\dot{W} \leq-0.5\left(x_{1}^{2}+x_{2}^{2}\right)+x_{2}^{2}+u^{2}
\end{gathered}
$$

with output $y=x_{2}\left(\sigma_{1}(s)=\sigma_{2}(s)=s^{2}\right)$. Then control $u=-k_{1}(\mathbf{x})+k_{2}(\mathbf{x})$ with $k_{1}(\mathbf{x})=a x_{2}, a>0.5$ and $k_{2}(\mathbf{x})=K \operatorname{sign}\left(x_{2}\right)$ admits all condition of Theorem 3 with $\lambda(s)=a s, \kappa(s)=(a-0.5) s^{2}, \mu(s)=0.5 s^{2}$. All functions $\sigma_{2}, \sigma_{1} \circ \lambda$ and $\kappa$ are square-law and, hence,

$$
\lim _{s \rightarrow+\infty} \frac{\sigma_{2}(s)+\sigma_{1} \circ \lambda(s)}{\kappa(s)}<+\infty ;
$$

inequality $x_{2} k_{2}(\mathbf{x})>x_{2} k_{1}(\mathbf{x})$ holds for $0<\left|x_{2}\right|<Y, Y=K / a$. This system is ISS for control $u=-k_{1}(\mathbf{x})+d$ with ISS Lyapunov function:

$$
\begin{gathered}
U(\mathbf{x})=W(\mathbf{x})+\frac{1+2 a^{2}}{a-0.5} V(\mathbf{x}), \\
\dot{U} \leq-0.5\left(x_{1}^{2}+x_{2}^{2}\right)+\left(2+\frac{0.5+a^{2}}{a-0.5}\right) d^{2} .
\end{gathered}
$$

Then set

$$
\Omega=\left\{\mathrm{x}: K / a \leq|\mathrm{x}| \leq \sqrt{1+\frac{1.5 a-0.75}{a^{2}+0.5}} \sqrt{4+\frac{1+2 a^{2}}{a-0.5}} K\right\}
$$

is always nonempty. Simulation results and bounds of set $\Omega$ are shown in Figure 4 for $a=1$ and $K=1 / 3$. 


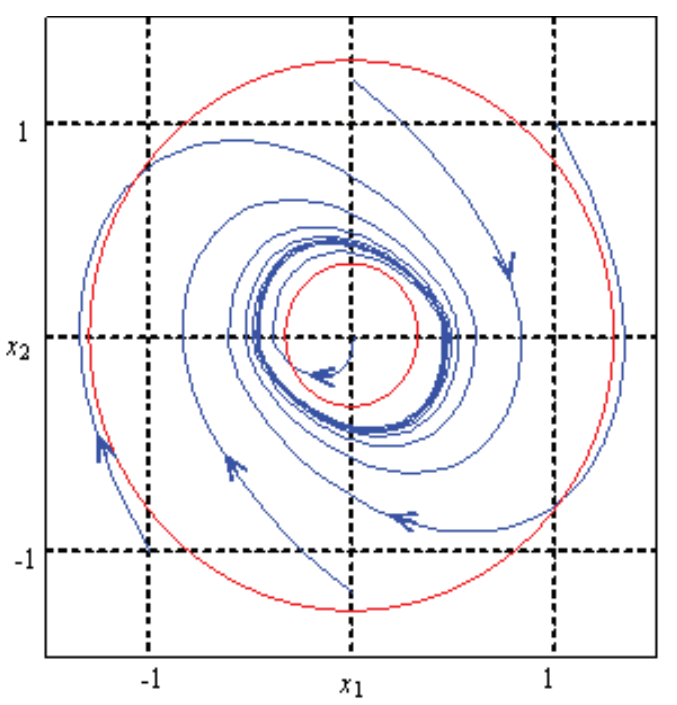

FIG. 4. Trajectories of linear oscillator under nonlinear feedback.

Based on the results of Theorem 3 and Corollary 2 it is possible to obtain the estimates of excitation indices of closed loop system for the case of nonvanishing signal $\mathbf{d}$.

Corollary 3. Let all conditions of Theorem 3 hold. Then for $\|\mathbf{d}\| \leq \gamma<+\infty$

$$
0 \leq \chi_{V}^{-}(\gamma) \leq \chi_{V}^{+}(\gamma) \leq \bar{\alpha} \circ \alpha_{4}^{-1} \circ \alpha_{5} \circ \alpha_{6}^{-1} \circ \delta(K+\gamma),
$$

if additionally

$$
\mathbf{y}(t)^{T} \mathbf{d}(t) \geq 0 \text { for all } t \geq 0,(3)
$$

then

$$
\underline{\alpha}(Y) \leq \chi_{V}^{-}(\gamma)<\chi_{V}^{+}(\gamma) \leq \bar{\alpha} \circ \alpha_{4}^{-1} \circ \alpha_{5} \circ \alpha_{6}^{-1} \circ \delta(K+\gamma) .
$$

Proof. Upper estimate on excitation indices follows from ISS property of the system with respect to input $\tilde{\mathbf{d}}$ (asymptotic gain property in [25]). Now let us consider time derivative of storage function $V$ :

$$
\begin{aligned}
\dot{V} & =\mathbf{y}^{T}\left(-\mathbf{k}_{1}(\mathbf{x})+\mathbf{k}_{2}(\mathbf{x})+\mathbf{d}\right)-\beta(\mathbf{x}) \\
& \geq\left[\mathbf{y}^{T}\left(-\mathbf{k}_{1}(\mathbf{x})+\mathbf{k}_{2}(\mathbf{x})\right)-\beta(\mathbf{x})\right]+\mathbf{y}^{T} \mathbf{d} .
\end{aligned}
$$

From conditions of Theorem 3, the expression in square brackets is positive for $0<$ $|\mathbf{y}|<Y<+\infty$, but the presence of sign-varying term $\mathbf{y}^{T} \mathbf{d}$ allows one to claim only $0 \leq \chi_{V}^{-}(\gamma) \leq \chi_{V}^{+}(\gamma)$ in common case. But if $\mathbf{y}(t)^{T} \mathbf{d}(t) \geq 0$ for all $t \geq 0$, then

$$
\begin{aligned}
& {\left[\mathbf{y}^{T}\left(-\mathbf{k}_{1}(\mathbf{x})+\mathbf{k}_{2}(\mathbf{x})\right)-\beta(\mathbf{x})\right]+\mathbf{y}^{T} \mathbf{d}} \\
& \quad \geq \mathbf{y}^{T}\left(-\mathbf{k}_{1}(\mathbf{x})+\mathbf{k}_{2}(\mathbf{x})\right)-\beta(\mathbf{x}),
\end{aligned}
$$

and the desired result follows by the same line of consideration as in Theorem 3 . Further let us suppose that it is possible a situation $\chi_{V}^{-}(\gamma)=\chi_{V}^{+}(\gamma)$ for some $\gamma$. But according to Definition 4, excitation indices admit conditions:

$$
\gamma_{1} \leq \gamma_{2} \quad \Rightarrow \quad \chi_{V}^{-}\left(\gamma_{2}\right) \leq \chi_{V}^{-}\left(\gamma_{1}\right) \text { and } \chi_{V}^{+}\left(\gamma_{1}\right) \leq \chi_{V}^{+}\left(\gamma_{2}\right)
$$


Applying the same arguments as in Corollary 2 for the results of Theorem 3 it is possible to obtain

$$
0<\chi_{V}^{+}(0)-\chi_{V}^{-}(0) \leq \bar{\alpha} \circ \alpha_{4}^{-1} \circ \alpha_{5} \circ \alpha_{6}^{-1} \circ \delta(K)-\underline{\alpha}(Y),
$$

therefore, $\chi_{V}^{+}(\gamma)-\chi_{V}^{-}(\gamma)>0$ for any $\gamma \geq 0$.

According to the corollary index $\chi_{V}^{+}(\gamma)$ is always bounded, that is more, it can not be equal to $\chi_{V}^{-}(\gamma)$ for any $\gamma \in R_{+}$with (3). Thus, system can not lose its oscillation ability for any large enough input disturbance possessing "coordination" condition (3) and such input $\mathbf{d}$ does not provide new equilibrium points into set $\Omega=\left\{V(\mathbf{x}): \underline{\alpha}(Y) \leq V(\mathbf{x}) \leq \bar{\alpha} \circ \alpha_{4}^{-1} \circ \alpha_{5}(K+\gamma)\right\}$ for system $\dot{\mathbf{x}}=\mathbf{f}\left(\mathbf{x}, \mathbf{k}_{1}(\mathbf{x})+\right.$ $\left.\mathbf{k}_{2}(\mathbf{x})+\mathbf{d}\right)$. Also it is worth to note, that the requirement (3) can be satisfied for $t \geq T$ only, where $0 \leq T<+\infty$.

5. Conclusion. In this paper conditions for oscillatority in the sense of Yakubovich applicable to nonlinear systems are proposed. Upper and lower bounds for oscillation amplitude are evaluated. Presented conditions are also necessary for some special class of uniformly oscillating systems. Relation between the oscillatority bounds and excitability indices for the systems with input is established. An important advantage of the results of the paper is their applicability to complex nonperiodic (e.g., chaotic) oscillations. Such an advantage is achieved due to using the concept of oscillatority in the sense of Yakubovich as the starting point of the whole study. The results are illustrated by examples: Evaluation of oscillations for Van der Pol and Hindmarsh-Rose neuron systems. As a side result a smooth nonquadratic Lyapunov function providing boundedness of Van der Pol system solutions has been found.

\section{Appendix.}

LEMMA A.1. Let system (1) have IOSS Lyapunov function $W$ and h-dissipative storage function $V$ as in Definition 2. If

$$
\lim _{s \rightarrow+\infty} \frac{\sigma_{2}(s)}{\alpha(s)}<+\infty,
$$

then system (1) is ISS with ISS Lyapunov function

$$
\begin{gathered}
U(\mathbf{x})=V(\mathbf{x})+\tilde{W}(\mathbf{x}), \quad \tilde{W}(\mathbf{x})=\rho(W(\mathbf{x})), \\
\rho(r)=\int_{0}^{r} q(s) d s, \quad q(s)=\frac{\alpha \circ \sigma_{2}^{-1}\left(0.25 \alpha_{3} \circ \alpha_{2}^{-1}(s)\right)}{1+0.5 \alpha_{3} \circ \alpha_{2}^{-1}(s)}, \\
\alpha_{4}(s)=\rho \circ \alpha_{1}(s), \quad \alpha_{5}(s)=\bar{\alpha}(s)+\rho \circ \alpha_{2}(s), \quad \alpha_{6}(s)=0.5 q\left(\alpha_{1}(s)\right) \alpha_{3}(s), \\
L_{\mathbf{f}(\mathbf{x}, \mathbf{u})} U(\mathbf{x}) \leq-\alpha_{6}(|\mathbf{x}|)+\delta(|\mathbf{u}|), \quad \delta(s)=\sigma(s)+2 \chi\left(2 \sigma_{1}(s)\right) \sigma_{1}(s), \\
\chi\left(2 \sigma_{2}(s)\right)=\alpha(s)\left[1+2 \sigma_{2}(s)\right]^{-1} .
\end{gathered}
$$

Proof. According to conditions of the lemma and Definition 2, the following series of inequalities holds for all $\mathbf{x} \in R^{n}$ and $\mathbf{u} \in R^{m}$ :

$$
\begin{gathered}
\alpha_{1}(|\mathbf{x}|) \leq W(\mathbf{x}) \leq \alpha_{2}(|\mathbf{x}|) ; \quad L_{\mathbf{f}(\mathbf{x}, \mathbf{u})} W(\mathbf{x}) \leq-\alpha_{3}(|\mathbf{x}|)+\sigma_{1}(|\mathbf{u}|)+\sigma_{2}(|\mathbf{y}|) \\
\underline{\alpha}(|\mathbf{y}|) \leq V(\mathbf{x}) \leq \bar{\alpha}(|\mathbf{x}|) ; \quad L_{\mathbf{f}(\mathbf{x}, \mathbf{u})} V(\mathbf{x}) \leq-\alpha(|\mathbf{y}|)+\sigma(|\mathbf{u}|),
\end{gathered}
$$

where $\alpha, \alpha_{1}, \alpha_{2}, \alpha_{3}, \underline{\alpha}, \bar{\alpha} \in K_{\infty}$ and $\sigma, \sigma_{1}, \sigma_{2} \in K$. Let us consider a new IOSS Lyapunov function

$$
\tilde{W}(\mathbf{x})=\rho(W(\mathbf{x})), \quad \rho(r)=\int_{0}^{r} q(s) d s,
$$

Copyright $@$ by SIAM. Unauthorized reproduction of this article is prohibited. 
where $q$ is some function from class $K$ (that will be defined later). Clearly function $\tilde{W}$ is again continuously differentiable, positive definite, and radially unbounded provided that $\rho \in K_{\infty}$. Its time derivative admits an estimate:

$$
L_{\mathbf{f}(\mathbf{x}, \mathbf{u})} \tilde{W}(\mathbf{x}) \leq q(W(\mathbf{x}))\left[-\alpha_{3}(|\mathbf{x}|)+\sigma_{1}(|\mathbf{u}|)+\sigma_{2}(|\mathbf{y}|)\right]
$$

To disclose the above inequality let us analyze consequently three situations:

(a) If $0.5 \alpha_{3}(|\mathbf{x}|) \geq \sigma_{1}(|\mathbf{u}|)+\sigma_{2}(|\mathbf{y}|)$, then

$$
L_{\mathbf{f}(\mathbf{x}, \mathbf{u})} \tilde{W}(\mathbf{x}) \leq-0.5 q(W(\mathbf{x})) \alpha_{3}(|\mathbf{x}|) ;
$$

(b) If $0.5 \alpha_{3}(|\mathbf{x}|)<\sigma_{1}(|\mathbf{u}|)+\sigma_{2}(|\mathbf{y}|)$ and $\sigma_{1}(|\mathbf{u}|) \leq \sigma_{2}(|\mathbf{y}|)$, then

$$
\begin{array}{r}
L_{\mathbf{f}(\mathbf{x}, \mathbf{u})} \tilde{W}(\mathbf{x}) \leq-q(W(\mathbf{x})) \alpha_{3}(|\mathbf{x}|)+2 q(W(\mathbf{x})) \sigma_{2}(|\mathbf{y}|) \\
\leq-q(W(\mathbf{x})) \alpha_{3}(|\mathbf{x}|)+2 \chi\left(2 \sigma_{2}(|\mathbf{y}|)\right) \sigma_{2}(|\mathbf{y}|)
\end{array}
$$

where $\chi(s)=q \circ \alpha_{2} \circ \alpha_{3}^{-1}(2 s)$;

(c) If $0.5 \alpha_{3}(|\mathbf{x}|)<\sigma_{1}(|\mathbf{u}|)+\sigma_{2}(|\mathbf{y}|)$ and $\sigma_{1}(|\mathbf{u}|)>\sigma_{2}(|\mathbf{y}|)$, then

$$
\begin{array}{r}
L_{\mathbf{f}(\mathbf{x}, \mathbf{u})} \tilde{W}(\mathbf{x}) \leq-q(W(\mathbf{x})) \alpha_{3}(|\mathbf{x}|)+2 q(W(\mathbf{x})) \sigma_{1}(|\mathbf{u}|) \\
\leq-q(W(\mathbf{x})) \alpha_{3}(|\mathbf{x}|)+2 \chi\left(2 \sigma_{1}(|\mathbf{u}|)\right) \sigma_{1}(|\mathbf{u}|)
\end{array}
$$

Thus, the time derivative of function $\tilde{W}$ calculated for system (1) can be rewritten in the form:

$$
\begin{aligned}
& L_{\mathbf{f}(\mathbf{x}, \mathbf{u})} \tilde{W}(\mathbf{x}) \leq-0.5 q(W(\mathbf{x})) \alpha_{3}(|\mathbf{x}|) \\
& \quad+2 \chi\left(2 \sigma_{2}(|\mathbf{y}|)\right) \sigma_{2}(|\mathbf{y}|)+2 \chi\left(2 \sigma_{1}(|\mathbf{u}|)\right) \sigma_{1}(|\mathbf{u}|) .
\end{aligned}
$$

Let function $\chi$ be taken to possess the following equality:

$$
\chi\left(2 \sigma_{2}(s)\right)=\frac{\alpha(s)}{1+2 \sigma_{2}(s)}
$$

such choice of $\chi$ is possible due to

$$
\lim _{s \rightarrow+\infty} \frac{\sigma_{2}(s)}{\alpha(s)}<+\infty
$$

with $q(s)=\frac{\alpha \circ \sigma_{2}^{-1}\left(0.25 \alpha_{3} \circ \alpha_{2}^{-1}(s)\right)}{1+0.5 \alpha_{3} \circ \alpha_{2}^{-1}(s)}$ from class $K$. Then system (1) is ISS with ISS Lyapunov function $U(\mathbf{x})=V(\mathbf{x})+\tilde{W}(\mathbf{x})\left(\alpha_{4}(s)=\rho \circ \alpha_{1}(s), \alpha_{5}(s)=\bar{\alpha}(s)+\rho \circ\right.$ $\alpha_{2}(s)$ ), indeed:

$$
\begin{aligned}
& L_{\mathbf{f}(\mathbf{x}, \mathbf{u})} U(\mathbf{x}) \leq-0.5 q(W(\mathbf{x})) \alpha_{3}(|\mathbf{x}|)+\sigma(|\mathbf{u}|) \\
& \quad+2 \chi\left(2 \sigma_{1}(|\mathbf{u}|)\right) \sigma_{1}(|\mathbf{u}|) \leq-\alpha_{6}(|\mathbf{x}|)+\delta(|\mathbf{u}|)
\end{aligned}
$$

where $\alpha_{6}(s)=0.5 q\left(\alpha_{1}(s)\right) \alpha_{3}(s)$ and $\delta(s)=\sigma(s)+2 \chi\left(2 \sigma_{1}(s)\right) \sigma_{1}(s)$. 
The next lemma is a corollary of Lemma A.1 presenting a variant of ISS stabilizing control law for a passive system.

Lemma A.2. Let system (1) be passive and IOSS in the sense of Definition 2 and

$$
\underline{\alpha}(|\mathbf{y}|) \leq V(\mathbf{x}) \leq \bar{\alpha}(|\mathbf{x}|), \quad \underline{\alpha}, \bar{\alpha} \in K_{\infty} .
$$

Then control

$$
\begin{gathered}
\mathbf{u}=-\mathbf{k}(\mathbf{x})+\mathbf{d}, \quad|\mathbf{k}(\mathbf{x})| \leq \lambda(|\mathbf{y}|), \quad \lambda \in K \\
\mathbf{y}^{T} \mathbf{k}(\mathbf{x})+\beta(\mathbf{x}) \geq \kappa(|\mathbf{y}|)+0.5|\mathbf{y}|^{2}, \quad \kappa \in K_{\infty} ; \\
\lim _{s \rightarrow+\infty} \frac{\sigma_{2}(s)+\sigma_{1} \circ \lambda(s)}{\kappa(s)}<+\infty
\end{gathered}
$$

where $\mathbf{d} \in R^{m}$ is new input (Lebesgue measurable and essentially bounded function of time), and provides for the system ISS property with ISS Lyapunov function:

$$
\begin{gathered}
U(\mathbf{x})=V(\mathbf{x})+\tilde{W}(\mathbf{x}), \quad \tilde{W}(\mathbf{x})=\rho(W(\mathbf{x})), \quad \rho(r)=\int_{0}^{r} q(s) d s, \\
q(s)=\frac{\kappa \circ \tilde{\sigma}_{2}^{-1}\left(0.25 \alpha_{3} \circ \alpha_{2}^{-1}(s)\right)}{1+0.5 \alpha_{3} \circ \alpha_{2}^{-1}(s)}, \quad \alpha_{4}(s)=\rho \circ \alpha_{1}(s), \\
\alpha_{5}(s)=\bar{\alpha}(s)+\rho \circ \alpha_{2}(s), \quad \alpha_{6}(s)=0.5 q\left(\alpha_{1}(s)\right) \alpha_{3}(s), \\
\delta(s)=0.5 s^{2}+2 \chi\left(2 \sigma_{1}(2 s)\right) \sigma_{1}(2 s) .
\end{gathered}
$$

Proof. From Definition 2 the following conditions hold for all $\mathbf{x} \in R^{n}$ and $\mathbf{u} \in R^{m}$ :

$$
\begin{gathered}
\alpha_{1}(|\mathbf{x}|) \leq W(\mathbf{x}) \leq \alpha_{2}(|\mathbf{x}|) \\
L_{\mathbf{f}(\mathbf{x}, \mathbf{u})} W(\mathbf{x}) \leq-\alpha_{3}(|\mathbf{x}|)+\sigma_{1}(|\mathbf{u}|)+\sigma_{2}(|\mathbf{y}|) \\
\underline{\alpha}(|\mathbf{y}|) \leq V(\mathbf{x}) \leq \bar{\alpha}(|\mathbf{x}|) ; \quad L_{\mathbf{f}(\mathbf{x}, \mathbf{u})} V(\mathbf{x}) \leq-\beta(|\mathbf{x}|)+\mathbf{y}^{T} \mathbf{u}
\end{gathered}
$$

with $\alpha_{1}, \alpha_{2}, \alpha_{3}, \underline{\alpha}, \bar{\alpha} \in K_{\infty}, \sigma_{1}, \sigma_{2} \in K$ and $\beta$ some nonnegative definite function. Substituting control in these inequalities, it is possible to obtain

$$
\begin{gathered}
L_{\mathbf{f}(\mathbf{x}, \mathbf{u})} W(\mathbf{x}) \leq-\alpha_{3}(|\mathbf{x}|)+\sigma_{1}(|\mathbf{d}-\mathbf{k}(\mathbf{x})|)+\sigma_{2}(|\mathbf{y}|) \\
\leq-\alpha_{3}(|\mathbf{x}|)+\sigma_{1}(2|\mathbf{d}|)+\sigma_{1}(2 \lambda(|\mathbf{y}|))+\sigma_{2}(|\mathbf{y}|) \\
L_{\mathbf{f}(\mathbf{x}, \mathbf{u})} V(\mathbf{x}) \leq-\beta(|\mathbf{x}|)+\mathbf{y}^{T}(\mathbf{d}-\mathbf{k}(\mathbf{x})) \leq-\kappa(|\mathbf{y}|)+0.5|\mathbf{d}|^{2}
\end{gathered}
$$

Thus, such control provides for closed loop system IOSS property and $h$-dissipativity with respect to new input $\mathbf{d}$. If

$$
\lim _{s \rightarrow+\infty} \frac{\tilde{\sigma}_{2}(s)}{\kappa(s)}<+\infty, \quad \tilde{\sigma}_{2}(s)=\sigma_{2}(s)+\sigma_{1} \circ \lambda(s),
$$

then all conditions of Lemma A1 are satisfied and the system is ISS with ISS Lyapunov function

$$
\begin{gathered}
U(\mathbf{x})=V(\mathbf{x})+\tilde{W}(\mathbf{x}), \quad \tilde{W}(\mathbf{x})=\rho(W(\mathbf{x})), \quad \rho(r)=\int_{0}^{r} q(s) d s \\
q(s)=\frac{\kappa \circ \tilde{\sigma}_{2}^{-1}\left(0.25 \alpha_{3} \circ \alpha_{2}^{-1}(s)\right)}{1+0.5 \alpha_{3} \circ \alpha_{2}^{-1}(s)}, \quad \alpha_{4}(s)=\rho \circ \alpha_{1}(s), \\
\alpha_{5}(s)=\bar{\alpha}(s)+\rho \circ \alpha_{2}(s), \quad \alpha_{6}(s)=0.5 q\left(\alpha_{1}(s)\right) \alpha_{3}(s), \\
\delta(s)=0.5 s^{2}+2 \chi\left(2 \sigma_{1}(2 s)\right) \sigma_{1}(2 s) .
\end{gathered}
$$

Copyright (c) by SIAM. Unauthorized reproduction of this article is prohibited. 


\section{REFERENCES}

[1] D. Angeli, Input-to-state stability of PD-controlled robotic systems, Automatica, 35 (1999), pp. $1285-1290$.

[2] D. Angeli, E.D. Sontag, And Y.A. Wang, A characterization of integral input to state stability, Systems Control Lett., 38 (1999), pp. 209-217.

[3] M. ArcaK And A. Teel, Input-to-state stability for a class of Lurie systems, Automatica, 38 (2002), pp. 1945-1949.

[4] V.I. Arnold, V.V. Kozlov, and A.I. Neishtadt, Mathematical Aspects of Classical and Celestial Mechanics, Springer Verlag, Berlin, 1997.

[5] N.N. Bogolyubov and Y.A. Mitropolsky, Asymptotic Methods in the Theory of Nonlinear Oscillations, Gordon and Breach, New York, 1962.

[6] Encyclopedia of Science and Technology, McGraw-Hill, 2005.

[7] A.L. FradKov, Exploring nonlinearity by feedback, Phys. D., 128 (1999), pp. 159-168.

[8] A.L. Fradkov, Investigation of physical systems by means of feedback, Automat. Remote Control, 60 (1999), pp. 3-22.

[9] V.N. Fomin, A.L. Fradkov, and V.A. Yakubovich, Adaptive Control of Dynamical Plants, Nauka, Moscow, 1981 (in Russian).

[10] A.L. Fradkov, I.V. Miroshnik, and V.O. Nikiforov, Nonlinear and Adaptive Control of Complex Systems, Kluwer Academic Publishers, 1999.

[11] A.L. Fradkov and A.Yu. Pogromsky, Introduction to Oscillations and Chaos, World Scientific, Singapore, 1998.

[12] C. Hayachi, Nonlinear Oscillations in Physical Systems, McGraw-Hill Book Company, New York, 1964.

[13] D. Hill and P. Moylan, Dissipative dynamical systems: Basic input - output and state properties, J. Franklin Inst., 309 (1980), pp. 327-357.

[14] J.L. Hindmarsh and R.M. Rose, A model of neunoral bursting using 3 coupled 1 st order differential-equations, Proc. R. Soc. Lond., B 221 (1984), pp. 87-102.

[15] Z.-P. Jiang, A. Teel, And L. Praly, Small - gain theorem for ISS systems and applications, Math. Control Signal Systems, 7 (1994), pp. 95-120.

[16] G.A. Leonov, I.M. Burkin, And A.I. Shepelyavyi, Frequency Methods in Oscillation Theory, Kluwer, Dordrecht, 1995 (in Russian: 1992).

[17] Y. Lin, E.D. Sontag, AND Y.A. WANG, Smooth converse Lyapunov theorem for robust stability, SIAM J. Control Optim., 34 (1996), pp. 124-160.

[18] J. Mallet-Paret and G.R. Sell, The Poincaré-Bendixson Theorem for monotone cyclic feedback systems with delay, J. Differential Equations, 125 (1996), pp. 441-489.

[19] S. Martinez, J. Cortes, And F. Bullo, Analysis and design of oscillatory control systems, IEEE Trans. Automat. Control, 48 (2003), pp. 1164-1177.

[20] V.V. Nemytski and V.V. Stepanov, Qualitative Theory of Differential Equations, Dover, New York, 1989.

[21] E.D. SontaG, Smooth stabilization implies coprime factorization, IEEE Trans. Automat. Control, 34 (1989), pp. 435-443.

[22] E.D. SontaG, Asymptotic amplitudes and Cauchy gains: A small gain principle and an application to inhibitory biological feedback, Systems Control Lett., 47 (2002), pp. 167-179.

[23] E.D. Sontag AND Y. WANG, Various results concerning set input-to-state stability, Proc. IEEE CDC 95, IEEE Publications, 1995, pp. 1330-1335.

[24] E.D. Sontag And Y. Wang, Notions of input to output stability, Systems Control Lett., 38 (1999), pp. 235-248.

[25] E.D. Sontag And Y. WANG, New characterization of the input to state stability property, IEEE Trans. Automat. Control, 41 (1996), pp. 1283-1294.

[26] E.D. SontAG AND Y. WANG, Output-to-state stability and detectability of nonlinear systems, Systems Control Lett., 29 (1997), pp. 279-290.

[27] R. Sepulchre, M. Janković, and P.V. Kokotović, Constructive Nonlinear Control, Springer-Verlag, New York, 1996.

[28] G.-B. Stan and R. Sepulchre, Global analysis of limit cycles in networks of oscillators, Proc. 5th IFAC Symposium on Nonlinear Control System (NOLCOS'04), 2004, Stuttgart, pp. 1433-1438.

[29] Wikipedia, the Free Encyclopedia, http://en.wikipedia.org/wiki/Oscillation_(mathematics), 2000.

[30] J.C. Willems, Dissipative dynamical systems - part I: General theory, Arch. Ration. Mech. Anal., 45 (1972), pp. 321-351.

Copyright (C) by SIAM. Unauthorized reproduction of this article is prohibited. 
[31] V.A. YAKubovich, Frequency oscillations conditions in nonlinear systems with stationary single nonlinearity, Siberian Math J., 14 (1973).

[32] V.A. YaKUBOVICH, Oscillations in systems with discontinuous and hysteresis nonlinearities, Autom. Remote Control, 12 (1975).

[33] V.A. Yakubovich and E.A. Tomberg, Conditions for self-induced oscillations in nonlinear systems, Siberian Math. J., 30 (1989), pp. 641-653.

Copyright (c) by SIAM. Unauthorized reproduction of this article is prohibited. 